\title{
Phytopathology
}

\section{Twenty-Five Years of the Binary Power Law for Characterizing Heterogeneity of Disease Incidence}

\author{
L. V. Madden, ${ }^{\dagger}$ G. Hughes, W. Bucker Moraes, X.-M. Xu, and W. W. Turechek
}

First and third authors: Department of Plant Pathology, Ohio State University, Wooster 44691; second author: Crop and Soil Systems Research Group, SRUC, King's Buildings, Edinburgh EH9 3JG, UK; fourth author: NIAB, East Malling Research, New Road, East Malling, ME19 6BJ, UK; and fifth author: United States Department of Agriculture-Agricultural Research Service, U.S. Horticultural Research Laboratory, 2001 South Rock Road, Ft. Pierce, FL 34945.

Accepted for publication 13 November 2017.

\begin{abstract}
Spatial pattern, an important epidemiological property of plant diseases, can be quantified at different scales using a range of methods. The spatial heterogeneity (or overdispersion) of disease incidence among sampling units is an especially important measure of small-scale pattern. As an alternative to Taylor's power law for the heterogeneity of counts with no upper bound, the binary power law (BPL) was proposed in 1992 as a model to represent the heterogeneity of disease incidence (number of plant units diseased out of $n$ observed in each sampling unit, or the proportion diseased in each sampling unit). With the BPL, the log of the observed variance is a linear function of the log of the variance for a binomial (i.e., random) distribution. Over the last quarter century, the BPL has contributed to both theory and multiple applications in the study of heterogeneity of disease incidence. In this article, we discuss properties of the BPL and use it to develop a general conceptualization of the dynamics of spatial heterogeneity in epidemics; review the use of the BPL in empirical and theoretical studies; present a synthesis of parameter estimates from over 200 published BPL analyses from a wide range of diseases and crops; discuss model fitting methods, and applications in sampling, data analysis, and prediction; and make recommendations on reporting results to improve interpretation. In a review of the literature, the BPL provided a very good fit to heterogeneity data in most publications. Eighty percent of estimated slope $(b)$ values from field studies were between 1.06 and 1.51 , with $b$ positively correlated with the BPL intercept parameter. Stochastic simulations show that the BPL is generally consistent with spatiotemporal epidemiological processes and holds whenever there is a positive correlation of disease status of individuals composing sampling units.
\end{abstract}

"The analysis of spatial pattern has become a fundamental part of science and a major tool of geographical and ecological analysis; its popularity has rapidly accelerated as computational resources for conducting spatial analysis have become widely available" (Rosenberg and Anderson 2016). In plant pathology, spatial pattern concerns the arrangement of diseased entities - such as diseased plants, leaves, or fruit-relative to each other and to their physical or biological surroundings (Campbell and Madden 1990; Gilligan 1982). In other words, "pattern is used to describe the dispersion in

\section{${ }^{\dagger}$ Corresponding author: L. V. Madden; E-mail: madden.1@osu.edu}

L. V. Madden was supported by state and federal funds appropriated to the Ohio Agricultural Research and Development Center. SRUC receives grant-in-aid from the Scottish Government.

*The $\boldsymbol{e}$-Xtra logo stands for "electronic extra" and indicates that one supplementary table is published online.

This article is in the public domain and not copyrightable. It may be freely reprinted with customary crediting of the source. The American Phytopathological Society, 2018. space of pathogens, diseased or infected plants or other components of pathosystems" (Madden et al. 2007). There has long been considerable interest in analyzing the arrangements of diseased individuals in botanical epidemiology (Hughes et al. 1997b; Jeger et al. 2017), to describe the observed patterns, to test whether the patterns are different from random (suitably defined), and to understand the processes generating the realized patterns.

There are many methods for quantifying spatial patterns of organisms. The approaches depend on, among other things, the data collection method (e.g., intensive mapping with explicit spatial referencing of the sampling units, or sparse sampling without explicit referencing of sampling units) and the nature of the diseased variable measured or observed (Hughes et al. 1997b). In terms of the latter situation, spatial methods are available for discrete random variables such as counts of diseased plants or lesions, which are not available for continuous random variables such as proportion of leaf area covered by lesions (disease severity) (Madden et al. 2007). The spatial heterogeneity (variability) in counts of individuals (such as diseased plants) among sampling units is an especially important measure of small-scale spatial pattern (i.e., at the scale of the sampling unit and smaller) (Dutilleul 2011; Madden 1989). Other 
measures are appropriate for characterizing larger-scale spatial patterns (Madden et al. 2007), which are not considered in this review.

In a seminal publication, Taylor (1961) showed with empirical data that the variance of the counts of organisms or other entities in sampling units $(V)$ is proportional to a power of the mean count per sampling unit $(m)$. He proposed the model $V=\kappa m^{b}$, where $\kappa$ and $b$ are parameters, with the scaling parameter $b$ sometimes known as a dispersion index or index of aggregation (a measure of spatial pattern). Below, we discuss how this power law can be viewed as a relationship between two variances. With a collection of datasets (for example, from different fields or different times in the same field), each characterized by a variance and a mean, one obtains a straight line with a slope of $b$ when $V$ is plotted against $m$ with logarithmic scales on both axes. Southwood (1966) may have been the first to refer to this model as Taylor's power law; most researchers now use this or some variation of this label.

Taylor was not the first to show a relationship between observed variances and means of counts but he was the first to advocate in a systematic way that the realized relationship was an ecological property of organisms. Before 1961, interest was on statistical issues such as the best method to transform counts in order to obtain a (nearly) constant variance (Bartlett 1936; Bliss 1941). There have now been thousands of datasets analyzed that demonstrate Taylor's power law. Although ecological problems have received the most attention, other studies have shown that Taylor's power law applies to numerous other systems, including clustering of genomic singlenucleotide polymorphisms, organ blood flow, human hematogenous cancer metastases, the New York Stock Exchange, and traffic in transportation networks (Fronczak and Fronczak 2010; Kendal 2004). Mechanisms generating Taylor's power law remain controversial, as discussed below.

Although Taylor's power law has been successfully applied in plant pathology (Gent et al. 2012; Van der Heyden et al. 2014), it was noted as early as 1987 that there were serious problems when Taylor's power law was applied indiscriminately to disease incidence datasets (Madden 1989; Madden et al. 1987). For instance, with Taylor's power law, the variance must increase monotonically with the mean, with no upper limit. However, it was noted that the variance of disease incidence first increased, reached a maximum, and then declined with the mean if mean incidence reached high enough levels (see Figure 4A in Madden et al. 1987). Examples are given in Figures 1A and B and 2A and B (explained below). This led Madden (1989) to conclude: "the appropriateness of Taylor's power law to plant pathogens is not clear." At the same time, studies in crop loss assessment sought ways to incorporate the effects of spatially heterogeneous pathogen injury when measured on a proportion scale in models of crop yield reduction (Hughes 1990; Hughes et al. 1989).

These strands came together at the VI International Workshop on Plant Disease Epidemiology, hosted by Professor Jürgen Kranz at Schloss Rauischholzhausen, Giessen, 3 to 9 September 1990. Subsequently, Hughes and Madden (1992) developed an alternative for the Taylor power law for characterizing spatial heterogeneity of disease incidence (or any count variable with an upper bound). The model, which can be considered as a relationship between two suitably defined variances, is now generally known as the binary power law (BPL). Over the past quarter-century, the BPL has been successfully used by several researchers to represent heterogeneity of disease incidence and some other incidence-type variables (counts with upper bounds). In this article, we review the development, interpretation, and applications of the BPL in plant pathology, and use the BPL to develop a general conceptualization of the dynamics of spatial heterogeneity in epidemics. We further present a synopsis of the results from the literature, point out sources of confusion regarding BPL analyses, and discuss different model-fitting methods and mechanisms that could generate the BPL.

\section{SPATIAL HETEROGENEITY}

A single sample of counts with an upper bound. We first consider heterogeneity in a single dataset (e.g., a field at a single time), and then expand to consider the analysis of multiple datasets using the BPL. Let $X$ represent the number of diseased individuals (plants, leaves, and so on) and $x$ the proportion of diseased individuals. If there are $n$ individuals in a sampling unit (e.g., quadrat, tree, or plant), then $X$ can range from 0 to a maximum of $n$ for any single sampling unit, and $x=(=X / n)$ can range from $0(=0 / n)$ to $1(=n / n)$. For instance, if one had a cluster sample (Hughes et al. 1996) of $N=100$ sampling units in the data set, with $n=10$ plants in each sampling unit (cluster), then $X$ for any sampling unit could take on integer values between 0 and 10 diseased plants, and $x$ could take on the values $0,0.1,0.2, \ldots, 0.9,1$.

Let $m$ represent the expected value of $X[m=\mathrm{E}(X)]$ and $V$ the variance of $X\left[V=\operatorname{var}(X)=\mathrm{E}\left(\{X-m\}^{2}\right)\right]$. To distinguish this variance from an alternative discussed below, we often refer to this as the observed variance (sometimes written as $V_{o b s}$ ). The expected value can be written as $m=n p$, where $p$ is a parameter representing the probability (or expected probability) of an individual being diseased. When $n$ is constant for each sampling unit, the moment estimate of $p$ is

$$
\hat{p}=\frac{\sum X_{i}}{n N}=\frac{\sum x_{i}}{N}
$$

Here, the $i$ subscript is an index for identifying the sampling unit $(i=1,2, \ldots, N)$. Because $\hat{p}$ is a mean proportion, it is the same as the commonly reported disease incidence. The moment estimate of $V$ is:

$$
\hat{V}=S^{2}=\frac{\sum\left(X_{i}-n \hat{p}\right)^{2}}{N-1}
$$

The maximum-likelihood estimate of $V$ is obtained by replacing $N-1$ with $N$ in the denominator.

When dealing with proportions $(x)$, the observed variance $[\operatorname{var}(x)]$ is given by $v=V / n^{2}$ when $n$ is a constant. It is estimated as:

$$
\hat{v}=s^{2}=\frac{\sum\left(x_{i}-\hat{p}\right)^{2}}{N-1}
$$

When the number of individuals per sampling unit is not constant but varies with sampling unit $\left(n_{i}\right)$, then the estimates of $p$ and the observed variances are more complicated because the expected value $\left(n_{i} p\right)$ varies with the sampling unit. However, this can be easily handled using the formulae in Cochran (1977); details for analyzing disease incidence are given in section 9.4.9 of Madden et al. (2007). The variable- $n$ equations were used by Madden et al. (1995a) in their BPL analysis.

When the probability of an individual (e.g., plant) being diseased is constant, so that the disease status of an individual does not depend on the disease status of other individuals, the distribution of $X$ is binomial (Madden and Hughes 1995; Madden et al. 2007). A binomial distribution is often considered to represent a random spatial pattern (Hughes and Madden 1993), at least at the scale of the sampling unit or smaller. As is well known, the variance of a binomial distribution is:

$$
V_{b i n}=n p(1-p)
$$

or for the proportions,

$$
v_{b i n}=p(1-p) / n
$$

Note that the maximum value of the binomial variance occurs at $p=1 / 2$. Estimates of the binomial variances are given by: 


$$
\begin{gathered}
\hat{V}_{b i n}=n \hat{p}(1-\hat{p}) \\
\hat{v}_{b i n}=\hat{p}(1-\hat{p}) / n
\end{gathered}
$$

If the number of diseased individuals $(X)$ has a binomial distribution, then $V=V_{\text {bin }}\left(\right.$ or $\left.v=v_{\text {bin }}\right)$. That is, $\mathrm{E}\left(\{X-m\}^{2}\right)$ for a binomial distribution reduces to $V_{\text {bin }}$ in equation $3 \mathrm{a}$; in terms of estimates, general equation 2a for an estimated variance is the same as $\hat{V}_{b i n}$ of equation $4 \mathrm{a}$ (if the denominator in equation $2 \mathrm{a}$ is $N$ and not $N-1$ ).

Overdispersion. When the probability of an individual being diseased is not constant, then the distribution of $X$ is not binomial. It may have a $\beta$-binomial, logistic-normal-binomial, or some other discrete distribution (Hughes and Madden 1993; Kriss et al. 2012; Ridout et al. 1999). In fact, the BPL leads directly and analytically to the $\beta$-binomial as the distribution for the data (Hughes and Madden 1993; Madden and Hughes 1995), supporting the popular use of this distribution in spatial analysis (Madden et al. 2007). Based on theoretical work on exponential dispersion models by Jørgensen

\section{FIGURE 1}

Spatial heterogeneity of the incidence of citrus canker in Brazil (based on results published by Gottwald et al. [2007]). Each point represents the results from one orchard (with $T=203$ different orchards). A, Observed variance of the proportion diseased $(v)$ in relation to disease incidence $(p) ; \mathbf{B}, \ln (v)$ in relation to $\ln (p)$; and $\mathbf{C}, \ln (v)$ in relation to the $\log$ of the binomial variance, $\ln [p(1-p) / n]$, where $n$ is the number of trees per sampling unit $(n=9)$. The solid line is for the fitted binary power law and the dashed line is for the binomial (random) situation. Natural logs were used, although the original paper used base-10 logs. Data courtesy of Tim Gottwald.
(1997), the so-called simplex-binomial distribution may also be appropriate when the variance relationship is described by the BPL; however, the lack of closed form for the simplex-binomial will likely preclude its use for routine data analysis. In general, $V>V_{\text {bin }}$ for nonbinomial distributions, a situation known as overdispersion or extrabinomial variation. Overdispersion is considered a form of small-scale spatial aggregation of disease incidence (Madden et al. 2007, chapter 9). There also may be underdispersion, characterized by $V<V_{b i n}$, although this is much less common than overdispersion.

A useful measure of overdispersion is the index of dispersion, $D$, a concept going back to Fisher (1925):

$$
D=V / V_{b i n}=v / v_{b i n}
$$

In practice, one substitutes the variance estimates in the ratio formula for $D$ (Madden and Hughes 1995; Sun and Madden 1997). Values of $D>1$ indicate overdispersion, $D<1$ indicate underdispersion, and $D=1$ indicates a binomial distribution (randomness or homogeneity). Most disease incidence datasets
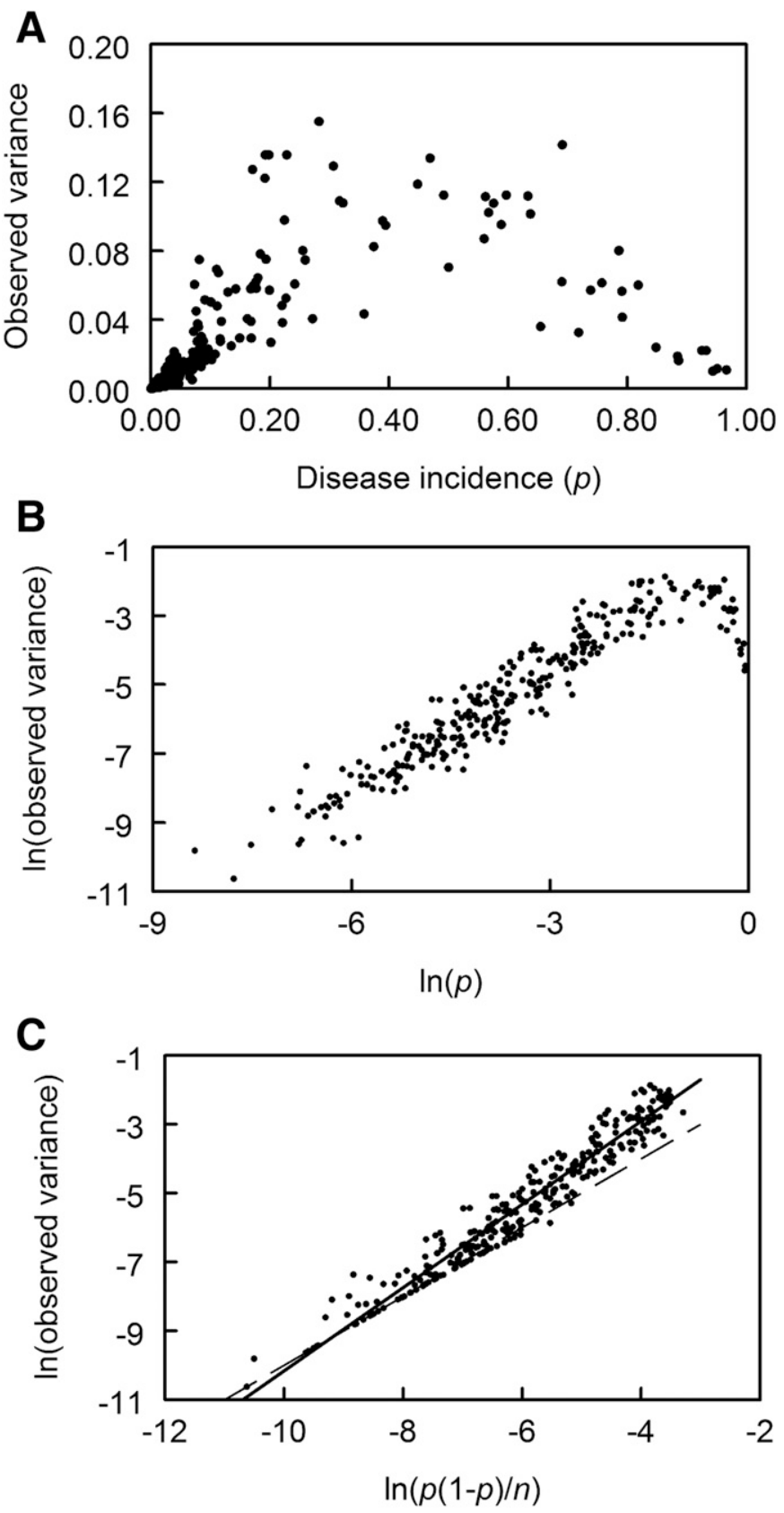
analyzed show some degree of overdispersion (Hughes et al. 1997b; Madden and Hughes 1995; Madden et al. 2007).

$D$ is greater than 1 when the probability of an individual being diseased is not constant (but a random variable) or when the disease status of an individual is positively correlated with the disease status of other individuals in the same sampling unit (Collett 2003; Ridout and Xu 2000; Ridout et al. 1999). Under quite general conditions, it can be shown that the observed variance equals:

$$
V=V_{b i n}[1+(\mathrm{n}-1) \rho]=[n p(1-p)][1+(n-1) \rho]
$$

or

$$
v=v_{b i n}[1+(\mathrm{n}-1) \rho]=[p(1-p) / n][1+(\mathrm{n}-1) \rho]
$$

where $\rho$ is the so-called intracluster correlation coefficient, a measure of the average correlation of the disease status of individuals within clusters (sampling units) (Hughes et al. 1996; Madden and Hughes $1995)$. It is also a parameterization of the aggregation parameter $(\theta)$ of the $\beta$-binomial distribution, although derivation of equations $6 a$ and $6 b$

FIGURE 2

Spatial heterogeneity of the incidence of potyvirus infection of tobacco in Kentucky (based on data published by Hughes and Madden [1992]). Each point represents a different time in the same epidemic (field code A-N-1984, with $T=17$ times). A, Observed variance of the proportion diseased $(v)$ in relation to disease incidence $(p) ; \mathbf{B}, \ln (v)$ in relation to $\ln (p)$; and $\mathbf{C}, \ln (v)$ in relation to the log of the binomial variance, $\ln [p(1-p) / n]$, where $n$ is the number of plants per sampling unit $(n=40)$.

The solid line is for the fitted binary power law and the dashed line is for the binomial (random) situation. Natural logs were used, although the original paper used base-10 logs. does not depend on the $\beta$-binomial distribution. The derivation of equations $6 \mathrm{a}$ and $6 \mathrm{~b}$ is explained thoroughly in sections 6.2 and 6.3 of Collett (2003). The factor $[1+(n-1) \rho]$ is equivalent to $D$ and to the design effect (deff) commonly used in survey sampling (Hughes and Madden 1995; Hughes et al. 1996).

The maximum value of $\rho$ is 1 , which gives the maximum value for the index of dispersion of $D=[1+n-1]=n$. When $\rho=0, D=[1+(n-1) \cdot 0]=1$ and, thus, $V=V_{\text {bin }}$ (equation 6a); this corresponds to independence of the disease status of individuals within a sampling unit (the so-called random distribution or homogeneity situation). The minimum $\rho$ is discussed later.

There are multiple ways of estimating $\rho$ (Hughes and Madden 1995; Hughes et al. 1996; Madden and Hughes 1995; Ridout et al. 1999). When $n$ is constant, a simple approach is based on the rearrangement of equations $6 \mathrm{a}$ or $6 \mathrm{~b}$ :

$$
\rho=(D-1) /(n-1)
$$

where $D$ is estimated using the estimated observed and binomial variances (equation 5).
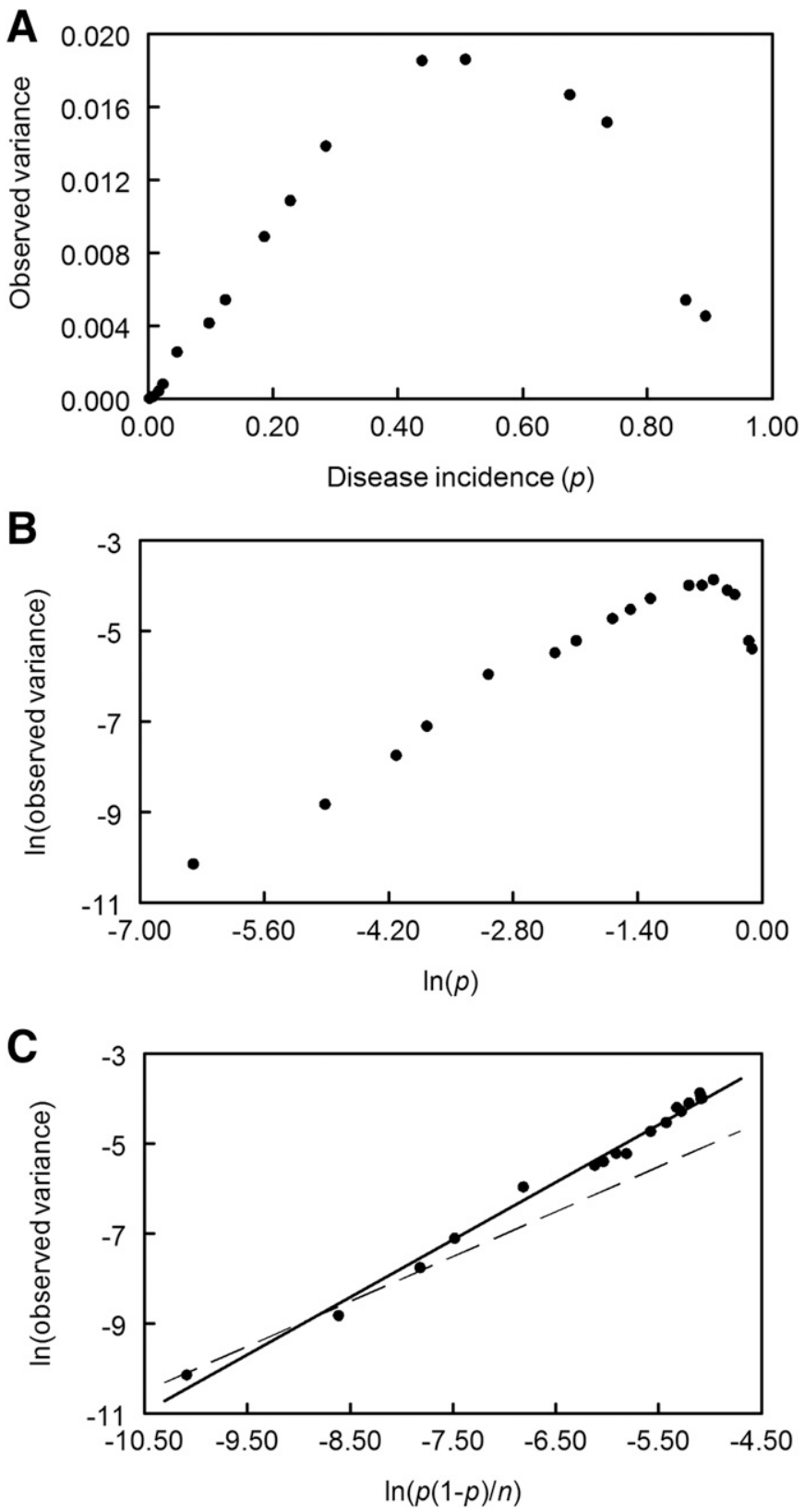
It should be noted that, in this article, the $p$ symbol indicates either the constant probability of disease (e.g., for a binomial distribution) or the expected (mean) probability of disease, the latter occurring when the probability of disease is a random variable (for instance, when $X$ has a $\beta$-binomial distribution). The distinction should be clear from context.

Comparison with unbounded count data. When $X$ has no upper bound, then the Poisson (not the binomial) is the discrete distribution for a random pattern (i.e., when the expected value is constant across all sampling units). In fact, the binomial distribution approaches the Poisson in the limit as $n$ increases indefinitely $(n \rightarrow \infty)$ while $m=n p$ is held constant (see section 9.6 in Madden et al. 2007). A property of the Poisson distribution is that the variance $\left(V_{P o i}\right)$ equals the mean; that is, $V_{P o i}=m$.

It is common to use the variance-to-mean ratio (VMR) to quantify overdispersion (and, hence, small-scale spatial aggregation) for a sample of unbounded counts; VMR $=V / m$ (with estimates substituted for the theoretical variance and mean). As pointed out by Patil and Stiteler (1974), because the variance of the Poisson distribution is the same as the mean, the VMR is really a version of index of dispersion $(D)$; that is, a ratio of two variances (one for the observed data and one that would correspond to a Poisson distribution). In particular, one can write $D=V / V_{P o i}$ for unbounded counts.

BPL. In contrast to Taylor's power law for counts with no upper bound, one may not observe a straight-line relationship when one plots the $\log$ of the estimated observed variance $(\hat{V}$ or $\hat{v})$ versus the $\log$ of the estimated incidence (given either as $\hat{p}$ or $n \hat{p}$ ) for a collection of samples (datasets) when the data have an upper bound of $n$ in each sampling unit (Madden 1989; Madden et al. 1987). As demonstrated in Figures $1 \mathrm{~A}$ and $\mathrm{B}$ and $2 \mathrm{~A}$ and $\mathrm{B}$, the observed variances increase monotonically at first, reach a maximum at approximately $\hat{p}=0.5$, and then decline at higher values of incidence. Of course, if there is a narrow range of mean incidence values across the samples, or if all the mean incidence values are $<0.5$ (or all $>0.5$ ), the increase and then decrease in variances would not be obvious. However, any model must take into account the empirical relationships shown in Figures 1 and 2.

Hughes and Madden (1992) developed a model for spatial heterogeneity of incidence data that takes into account the relationships shown in Figures 1 and 2 for the full range of incidence values. The model was based on the realization that the usual Taylor power law (for counts without an upper bound) can be viewed as a relationship between two variances, the observed variance and the variance the observations would have if they had a Poisson distribution (i.e., a random distribution). That is, one could write Taylor's law as $V=\kappa m^{b}=\kappa V_{P o i}^{b}$.

Thus, a power law for heterogeneity can be considered, more broadly, as a relationship between an observed variance and a variance for a suitably defined discrete distribution for a random pattern (at least at some spatial scale) (Hughes and Madden 1992; Madden and Hughes 1995). A general way of writing this is:

$$
V=\kappa V_{r a n}^{b}
$$

where $V_{\text {ran }}$ is the variance for the corresponding suitably defined random distribution. For discrete data with an upper bound of $n$, equation $8 \mathrm{a}$ is written explicitly as:

$$
V=\kappa V_{\text {bin }}^{b}
$$

because the binomial is the corresponding random distribution. It is not surprising, therefore, that there is not a straight-line relationship between the $\log$ of the variance and the $\log$ of the mean, because $\ln (m)$ $\left[=\ln \left(V_{P o i}\right)\right]$ is only directly relevant in this variance-variance context when the random variable has a Poisson distribution, and the Poisson is not generally appropriate for disease incidence. However, as a special case for equation $8 \mathrm{~b}$, it is interesting to note that, if $n$ is large and $p$ is very small, $1-p \approx 1$; this means that the binomial variance (equation 3a) is approximately given by $V_{\text {bin }} \approx n p \times 1=n p=m=$ $V_{P o i}$ at small $p$. (This also follows from the relationship between the Poisson and binomial distributions). Thus, in this scenario, Taylor's power law can be considered an approximation to the BPL for situations with low incidence.

The conceptualization in equation $8 \mathrm{a}$ and $8 \mathrm{~b}$ is supported by knowledge that the index of dispersion $D$ (or the VMR as a special case) is a ratio of two variances, one representing the observed variance and one representing the variance expected if the observations had a distribution consistent with a null hypothesis of randomness (Fisher 1925; Patil and Stiteler 1974). D is used as a summary measure from an individual data set (for example, for multiple quadrats in one field at one time), whereas the power law is used to summarize information for multiple data sets (i.e., different times, different fields, or both).

The Hughes and Madden (1992) model, often labeled the BPL or the binary form of Taylor's power law, can be written in several specific ways, all based on equation 8b. Depending on the formulation, different symbols are used for the scaling $\kappa$ parameter. For the bounded counts, one can write:

$$
V=A_{x}[n p(1-p)]^{b}
$$

And, for proportions, one can write:

$$
v=A_{p}[p(1-p) / n]^{b}
$$

When $n$ is constant, then equation $9 \mathrm{~b}$ can be equivalently written as $v=\left(A_{p} n^{-b}\right)[p(1-p)]^{b}$.

We discuss meaning of the $A_{X}$ or $A_{p}$ parameter below. Defining $a=A_{p} n^{-b}$, then one can write the proportion version of the BPL equivalently as:

$$
v=a[p(1-p)]^{b}
$$

In practice, one replaces the theoretical variances $\left(V, v, V_{b i n}\right.$, and $v_{\text {bin }}$ ) and $p$ by their estimates (see equations $2 \mathrm{a}, 2 \mathrm{~b}, 4 \mathrm{a}, 4 \mathrm{~b}$, and 1 ).

In general, we preserve the theoretical variance and $p$ variables in the presented models because we assume that the empirical relationships seen in graphs such as Figures 1, 2, and 3 represent relationships between the true observed and binomial variances. The model-fitting section below further addresses the issue of theoretical and estimated variances.

After taking logs, the three versions of the BPL can be written as:

$$
\begin{aligned}
\ln (V) & =\ln \left(A_{X}\right)+b \ln [n p(1-p)] \\
\ln (v) & =\ln \left(A_{p}\right)+b \ln [p(1-p) / n] \\
\ln (v) & =\ln (a)+b \ln [p(1-p)]
\end{aligned}
$$

That is, the $b$ exponent of the BPL becomes the slope of the straight-line relationship between the log of the observed variance and the $\log$ of the binomial variance (for either counts or proportions). This can be seen in Figures $1 \mathrm{C}$ and $2 \mathrm{C}$ for the two introductory examples. Figure 3 shows several more examples taken from the literature, demonstrating a representative range of results from diverse pathosystems (these were not chosen to be the best fits found in the literature but to demonstrate a diversity of 
situations, some of which are discussed later). Note that the farthest righthand side on the abscissa of these plots represents the $\log$ of the binomial variance at $p=1 / 2$ (the specific value depends on $n$ ). The farthest lefthand side on the abscissa represents the $\log$ of the binomial variance at $p$ close to 0 and close to 1 . The intercept is the $\log$ of either $A_{X}, A_{p}$, or $a$, depending on the model formulation.

All versions of the BPL based on equation $8 \mathrm{~b}$ are symmetrical about $p=1 / 2$; for instance, predicted $V$ at $p=0.1$ is the same as at $p=0.9$. A more complex model is needed to deal with general asymmetry in the $V: V_{\text {bin }}$ relationship. The " $a$ " version of the BPL provides a convenient way to deal with this problem. Equation $9 \mathrm{c}$ can be written as $v=a[p(1-p)]^{b}=a p^{b}(1-p)^{b}$.

This suggests a generalization where the exponent for $p$ is not the same as for $(1-p)$ :

$$
v=a p^{b_{1}}(1-p)^{b_{2}}
$$

where $b_{1}$ and $b_{2}$ are parameters. Log transformation leads to:
A

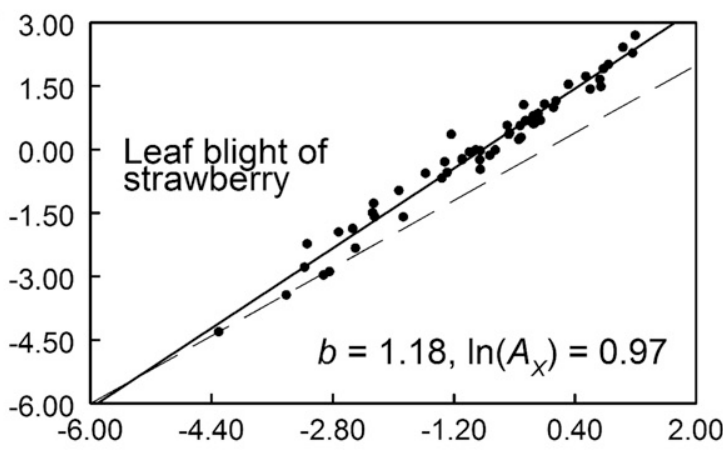

B

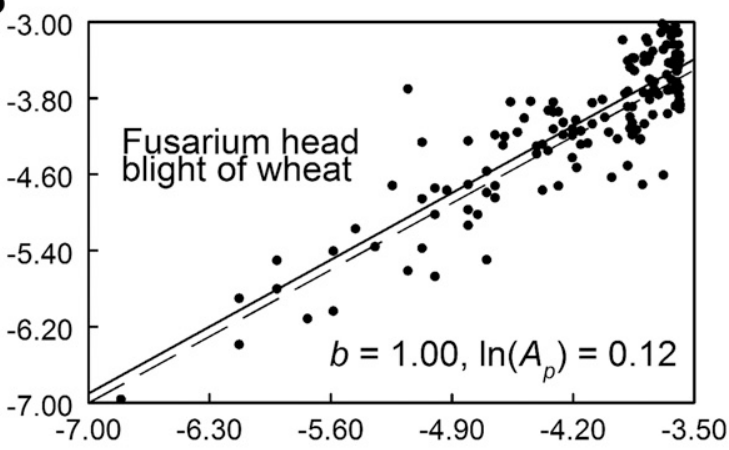

C

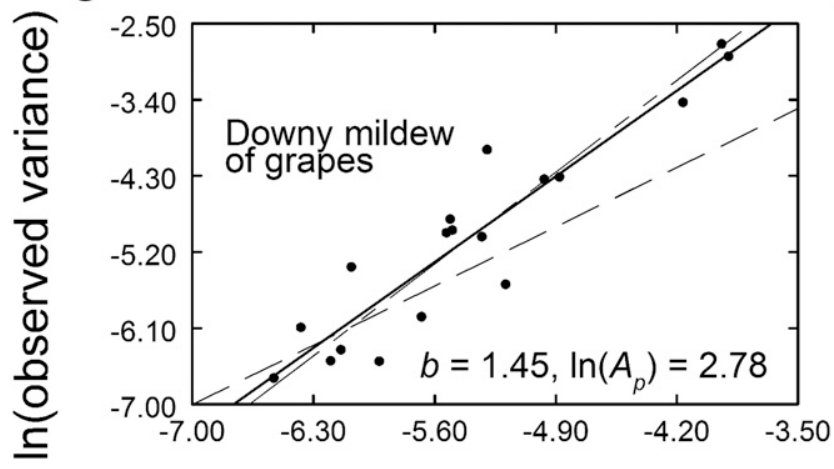

E

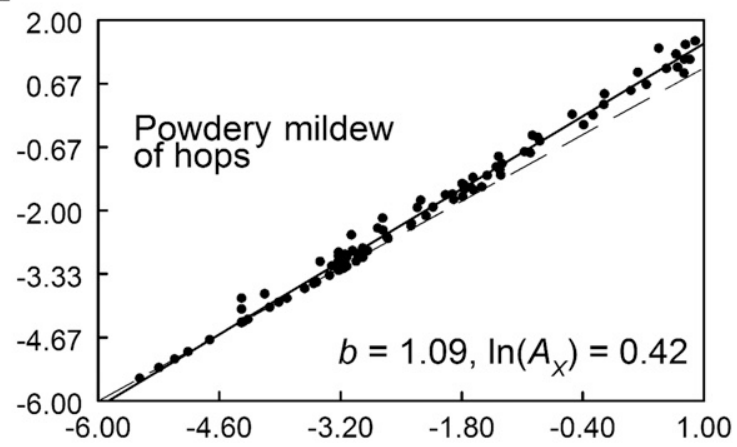

D

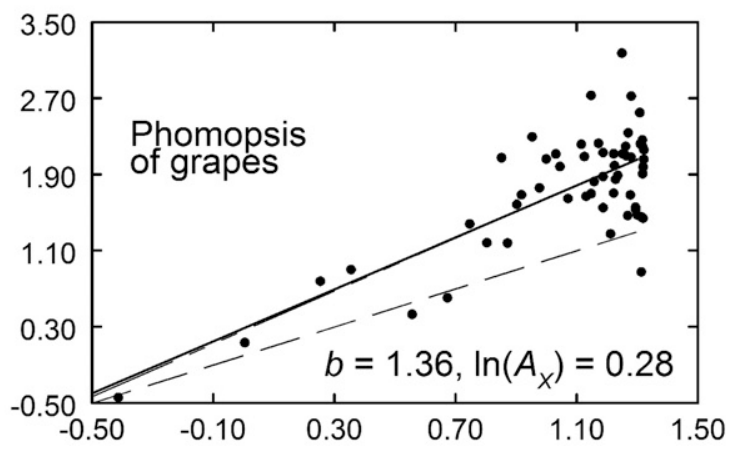

$\mathbf{F}$

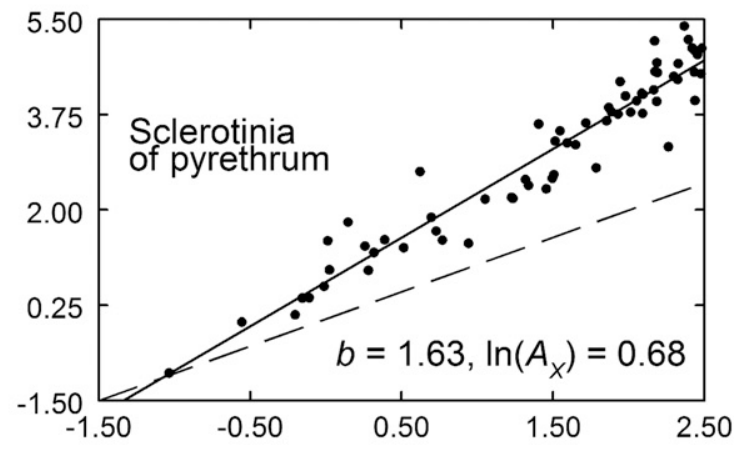

In(binomial variance)

\section{FIGURE 3}

Relationship between the log of the observed variance $[\ln (V)$ or $\ln (V)$ of disease incidence and the log of the binomial variance $\{\ln [p(1-p) / n]$ or $\ln [n p(1-p)]\}$ for six example published datasets, where $p$ is the probability of disease (incidence) and $n$ is the number of individuals in a sampling unit. A, Phomopsis leaf blight of strawberry (Turechek and Madden 1999a, year 1997 leaflet results). B, Fusarium head blight of wheat spikes (Spolti et al. 2015). C, Downy mildew of grape leaves (Madden et al. 1995a, year 1990b dataset). D, Phomopsis cane and leaf spot of grape (Nita et al. 2012; leaf data results). E, Powdery mildew of hops leaves (Gent et al. 2008, year 2000 leaf disease results). F, Sclerotinia flower blight of pyrethrum (Pethybridge et al. 2010). Examples used either the count version of the binary power law (equation 10a; graphs A, D, E, and F) or the proportion version (equation 10b; graphs B and C). Solid line is for the fitted binary power law and the dashed line is for the binomial (random) situation. Natural log used for all graphs, although some original papers used base-10 logs (e.g., graph C). Data courtesy of Sarah Pethybridge, David Gent, and Emerson del Ponte. 


$$
\ln (v)=\ln (a)+b_{1} \ln (p)+b_{2} \ln (1-p)
$$

For a symmetrical relationship, $b_{1}=b_{2}=b$. Hughes and Madden (1992) developed this model together with the symmetric versions of the BPL in their original article. Equation 10d has not seen much use since 1992, except in simulation studies (Xu and Madden 2014; $\mathrm{Xu}$ and Ridout 2000). Apparently, investigators are satisfied with the fit of the simpler two-parameter BPL model, or there is no strong evidence for asymmetry with empirical data sets. To detect asymmetry, a wide range of incidence values would be needed in a dataset. We do not further use equations $9 \mathrm{~d}$ and $10 \mathrm{~d}$ in this review.

We used natural (i.e., base $e$ ) $\log$ in equations $10 \mathrm{a}$ to $10 \mathrm{c}$, although $\log$ base 10 [i.e., $\log _{10}(\cdot)$ ] or any other base can be used. The choice of log base has no effect on the $b$ parameter, but it does affect the intercept in equations 10a to 10c. With knowledge of $n$ (for constant $n$ ) and $b$, the $\log$ base, and whether counts or proportions are being analyzed, one can convert between $A_{X}, A_{p}$, and $a$. For instance, after suitable back-transformation (antilog) of the intercepts of equations $10 \mathrm{a}$ to $10 \mathrm{c}$, one can show the following:

$$
\begin{aligned}
& a=A_{p} n^{-b}=A_{X} n^{b-2} \\
& A_{p}=A_{X} n^{2 b-2}
\end{aligned}
$$

With a variable $n$, one can use $\bar{n}$ in equations $11 \mathrm{a}$ and $\mathrm{b}$; however, the relationship among the parameters is then only approximate.

Use of the count with upper bound (equations 9a and 10a) or proportion (equations $9 \mathrm{~b}$ and $10 \mathrm{~b}$ or $10 \mathrm{~b}$ and $10 \mathrm{c}$ ) versions of the BPL is arbitrary. We have used both versions in different studies (Turechek and Madden 1999a,b for counts; Madden et al. 1995a for proportions). Because plant pathologists typically think of disease incidence as a proportion or percentage (Madden et al. 2007, chapter 2 ), it may be "natural" to work with the proportion version of the BPL. Approximately $60 \%$ of the published studies have utilized the proportion formulation (where it is possible to determine which version was used). Interestingly, it is very uncommon to use equations $9 \mathrm{c}$ and $10 \mathrm{c}$ (the " $a$ " parameter model version). However, the $a$ parameter (typically determined from $A_{p}$ or $A_{X}$ using equation 11a) is convenient to utilize for several applications (see below).

A diagram of different versions of the BPL and the relationship of BPL parameters to measures of overdispersion (to be discussed below) is given in Figure 4.

Interpretation of the parameters. Going back to the initial development of Taylor's power law (Taylor 1961), emphasis is usually placed on $b$ as a metric to characterize heterogeneity and, hence, pattern or aggregation at the small spatial scale (scale of the sampling unit or smaller). However, both $\kappa$ (i.e., $A_{X}, A_{p}$, or $a$ ) and $b$ need to be jointly considered to fully interpret the pattern (equations 9a to 9c). When $b=1$ and $A_{p}=1$ [or $\ln \left(A_{p}\right)=0$ ], then the BPL reduces to $v=v_{\text {bin }}$; that is, the observed variance equals the variance that the data would have if $X$ had a binomial distribution. We can also present this equivalently in terms of $b=1$ and $A_{X}=1$; for ease of presentation, we often stick with one BPL formulation (proportion or count) unless the distinction between formulations is needed for interpretation. The so-called binomial line on a log-log scale is usually presented in the BPL graph (Figs. 1C, 2C, and 3). If $b=1$ and $A_{p}>1$ [or $\ln \left(A_{p}\right)>0$ ], then there is overdispersion that does not depend on the level of incidence; the degree of heterogeneity is the same for all incidence values. A situation when $b$ is approximately equal to 1 is shown in Figure 3B. In this case, $\ln \left(A_{p}\right)$ is only slightly larger than 0 .

If $b$ does not equal 1 , then overdispersion (heterogeneity) systematically varies with the level of incidence. The actual value of $v$ may be less than, greater than, or the same as $v_{b i n}$ at any given value of incidence. Typically, $b>1$, which means that $v$ is increasing faster than $v_{\text {bin }}$ (the latter being a function of $p$ and $1-p$ ) (equation $3 b$ ). Typically, most (but not all) of the $v$ values are above the $v_{\text {bin }}$ binomial line in the publications using the BPL (e.g., Figs. 1C, 2C, and 3). The $v$ values sometimes cross below the binomial line at $p$ values close to 0 and 1 (near the far left abscissa). At such values of $v$, dispersion is typically indistinguishable from random.

Another way of intuitively "seeing" the effects of $b$ on heterogeneity is to reconsider the index of dispersion $(D$; equation $5)$ and intracluster correlation ( $\rho$; equation 6b) (Fig. 4). If one substitutes equation $9 \mathrm{~b}$ of the BPL for $v$ in the numerator and equation $3 \mathrm{~b}$ for $v_{\text {bin }}$ in the denominator, one obtains:

$$
D=\frac{A_{p}[p(1-p) / n]^{b}}{p(1-p) / n}=A_{p}[p(1-p) / n]^{\mathrm{b}-1}
$$

Whenever $b>1, D$ increases with the binomial variance; in contrast, when $b<1, D$ decreases with increases in the binomial variance. If $\left.b=1, D=A_{p}[p(1-p) / n)\right]^{0}=A_{p} 1=A_{p}$.

Equivalently, if one worked with the counts, $D=A_{X}$ when $b=1$. Thus, $A_{p}\left(\right.$ or $\left.A_{X}\right)$ is identical to $D$ when $b=1$, a measure of (fixed) overdispersion. If $A_{p}=A_{X}=b=1$, then $D=1$, as required. If $b>1$, then $D$ depends on $p$ (as well as on $n$ and $b$ ), and will have its largest value at $p=1 / 2$.

The intracluster correlation $(\rho)$ can be determined from the parameters of the BPL by rearranging equation $6 \mathrm{~b}$ (or $6 a$ ), after substituting equation $9 \mathrm{~b}$ or $9 \mathrm{c}$ for $v$. The version based on using the $a$ parameter formulation (equation 9c) is given by:

$$
\rho=\left(\frac{n}{n-1}\right)\left(\frac{a}{f(p)}-\frac{1}{n}\right)
$$

where $f(p)=[p(1-p)]^{1-b}$ (Hughes et al. 1996). If the $A_{X}$ or $A_{p}$ model versions are used (equations $9 \mathrm{a}$ or $9 \mathrm{~b}$, respectively), then one can utilize equation 11a to determine $a$ in order to apply this equation. Other references give algebraically equivalent expressions either for $\rho$ or for the $\beta$-binomial $\theta$ parameter $[\theta=\rho /(1-\rho)]$ (Madden et al. 2007, page 245; Turechek and Madden 1999a; Turechek and Mahaffee 2004), using either $a, A_{p}$, or $A_{X}$ (researchers need to be careful to use the appropriate equation because authors vary a lot in their notation for the "intercept" parameter in the BPL). When $b>1$, $\rho$ has a maximum at $p=1 / 2$, and declines to, or possibly below, 0 as $p$ approaches 0 or to 1 (Madden and Hughes 1995). When $b=1$, equation 13 reduces to the constant $(n a-1) /(n-1)$; a little algebra shows that this is equal to $\left(A_{p}-1\right) /(n-1)$ or $\left(A_{X}-1\right) /(n-1)$, which is the same as equation 7 based on $D$ (Fig. 4). When $A_{p}=A_{X}=b=1$, then $\rho=0$ (i.e., no overdispersion), as required.

The relationship between estimated $\rho$ and $p$ is demonstrated in Figure 5 for the tobacco virus disease data set in Figure 2. The BPL provides a good fit to the variance data (equation 10b), with an $R^{2}$ value of 0.99 , but the estimates of variances made from the data do not fall exactly on the $\log -\log \operatorname{line}$. Small variation in $\ln (v)$ at a given $p$ (or $v_{\text {bin }}$ ) can translate into much larger variation on the scale of $v$ and, hence, the scale of $\rho$ (or $D$ ) (Fig. 5A). Many published data sets show considerably larger variation in the plot of $\rho$ (or $D$ or $\theta$ ) versus disease incidence (Bassanezi et al. 2003; Dallot et al. 2003; Gent et al. 2006, 2008; Gigot et al. 2017; Gosme et al. 2007; Gottwald et al. 2007; Laranjeira et al. 2006; Madden et al. 1995a,b; NavasCortés et al. 2008; Nita et al. 2012; Savary et al. 2001; Spolti et al. 2015; Turechek and Mahaffee 2004; Turechek et al. 2014) because the $R^{2}$ for the fit of the BPL may be $<0.99$ (although still very high). With the scatter typically seen in the empirical $\rho: p$ graphs, it might be difficult for researchers to determine the underlying relationship between these parameters without relying on the BPL results. 
One challenge with graphs such as Figure 5 is that there are often several observations clustered near $p=0$, making it difficult to see the change in $\rho$ (or $D$ or $\theta$ ) with increasing $p$. Therefore, sometimes researchers use a log scale for $p$ (Fig. 5B) (Bassanezi et al. 2003; Gent et al. 2006; Turechek and Madden 1999a; Turechek and Mahaffee 2004). It is important to note that the log scale for the abscissa is not based on any underlying model or principle - if the BPL in equations 9 a to c holds, there is a symmetric relationship between $\rho$ (or $D$ or $\theta$ ) and $p$.

\section{SYNOPSIS OF THE PUBLICATIONS USING THE BPL}

Overview. The BPL has been used in at least 74 publications since it was introduced in Hughes and Madden (1992). Of these, 61 papers report on original BPL results for different crops and diseases, where a given paper could have results from one or multiple BPL analyses (different times, years, sampling conditions, or different diseases). Another four papers report on the proportion of plants infested by insects or on proportion results from ecological studies unrelated to plant diseases (Guan et al. 2016; Nault and Kennedy 1996; Shiyomi et al. 2001; Tsuiki et al. 2005). The remaining papers deal with theoretical research on the BPL (such as simulations) or applications based on (previously obtained) BPL results (e.g., development of sampling plans). Most of the published papers involve several spatial or temporal analyses in addition to the BPL analyses; spatial analysis may be for small-scale (BPL, discrete distributions, and overdispersion or aggregation metrics) and larger-scale patterns (semivariograms, SADIE [statistical analysis by distance indices], or autocorrelations) (Madden et al. 2007). It is difficult to determine the total number of investigations using the

FIGURE 4

Relationship between some different versions of the binary power law (BPL) and the relationship of the BPL parameters to the index of dispersion $(D)$ and the intracluster correlation $(\rho)$. Equation numbers are given in the text.

\footnotetext{
FIGURE 5

Relationship between the intracluster correlation $(\rho)$ and disease incidence $(p)$ for the tobacco potyvirus disease example in Figure 2 (Hughes and Madden 1992). Curve represents predicted $\rho$ based on equation 13 .

Righthand graph shows incidence on a log scale to better see the estimated and predicted
}

BPL because some papers do not cite the original Plant Pathology article (Hughes and Madden 1992) or the more comprehensive Annual Review of Phytopathology article (Madden and Hughes 1995). Labeling leads to other confusion. Some authors indicate that they used Taylor's power law but actually (and correctly) used the BPL for their incidence data. In at least one case, authors indicated that they used the BPL but actually used the Taylor power law.

The BPL has been used for diseases of over 25 crops for both annuals and (woody and herbaceous) perennials (Table 1). The sampling unit in each data set could be either natural (e.g., grape shoot, where all the leaves on the shoots are assessed for downy mildew symptoms) (Madden et al. 1995a), or arbitrary (dividing a lattice of trees in an orchard into $N$ clusters of size $n$ ) (Gottwald et al. 2007). In the latter case, the size of the sampling unit $(n)$ may be based on sampling practicalities or prior experience and, sometimes, more than one value of $n$ is considered. Citrus crops, strawberry, wheat, and hops have had the most BPL publications. Fungal-, oomycete-, viral-, bacterial-, and phytoplasma-caused diseases were the focus of $34,3,16,5$, and 2 papers, respectively. One paper dealt with diseases of unknown etiology. A given paper may have results for more than one disease. Results are summarized in Table 1 for papers that provided useable results for comparisons.

Challenges in interpreting the published literature. Authors do not always report all the relevant aspects of their investigation when using the BPL. This can require additional work to fully interpret the estimated parameter values and to compare results across studies; in some cases, it is not possible to use some of the published results in a comparative sense because of missing or misreported information. We describe some of the common problems here.

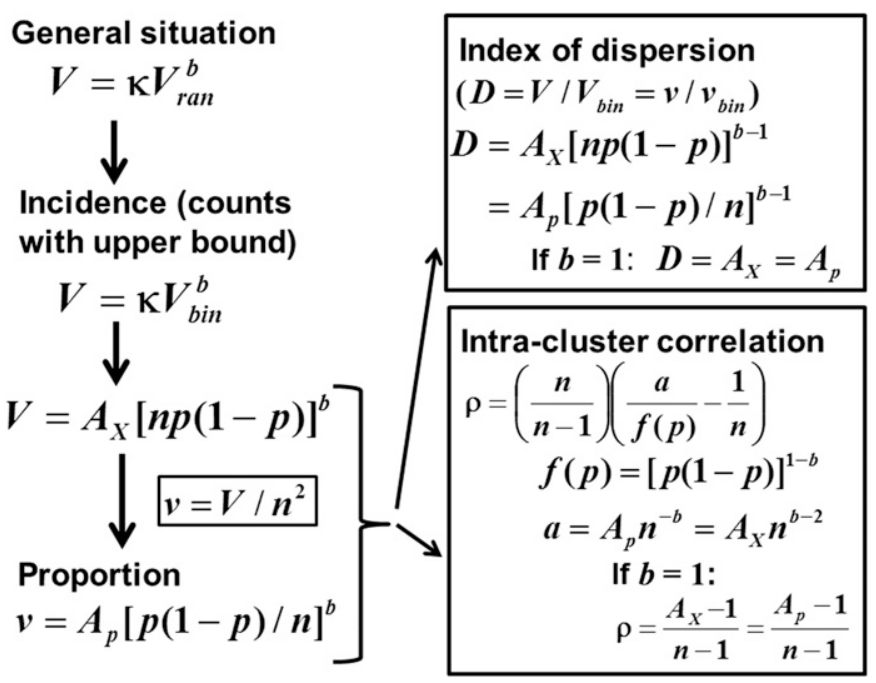

$\rho$ at low incidence (the transformation is not
based on theory).

$\rho$ at low incidence (the transformation is not
based on theory).
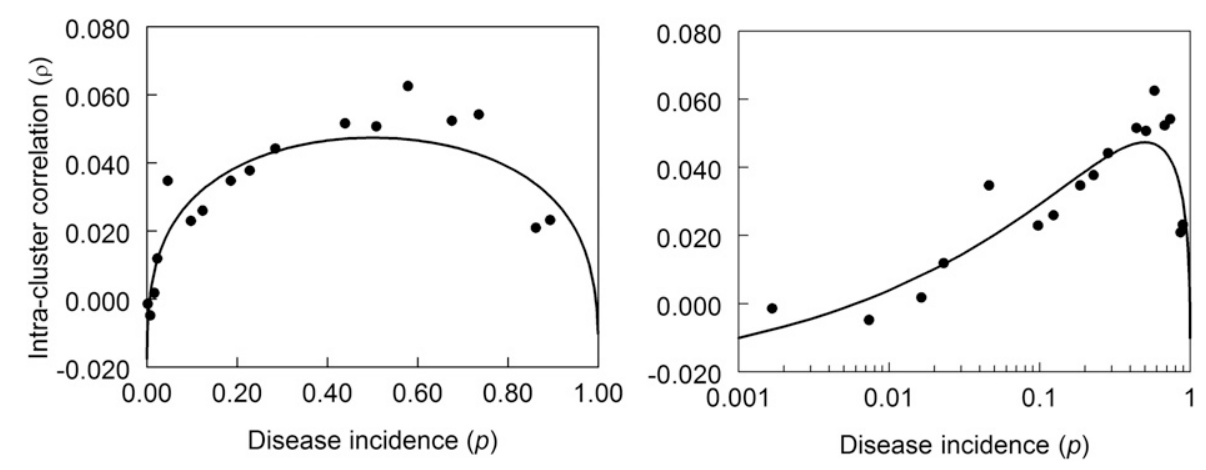
TABLE 1

Articles identified where the binary power law (BPL) (equations 10a to d) was used to characterize spatial heterogeneity (i.e., overdispersion) of disease incidence, and the range of estimated $a$ and $b$ parameters in each study (where a was determined directly or from $A_{X}$ or $A_{p}$ [equations $11 \mathrm{a}$ and $\mathrm{b}$ ], depending on the formulation of the BPL used by the authors) ${ }^{\mathrm{a}}$

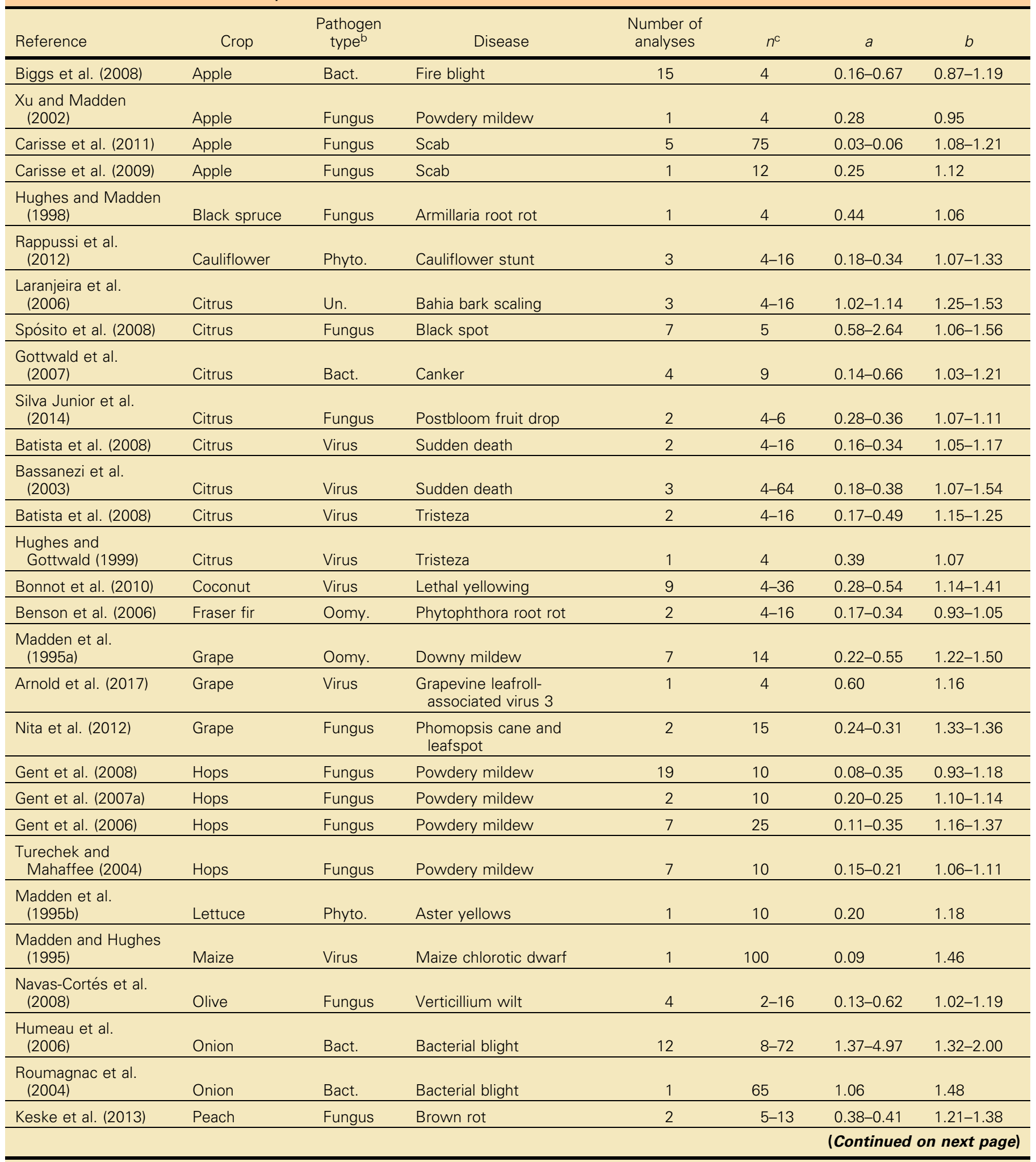

a Selection criteria are described in the text (articles are not included if sampling-unit size, $n$, was not identified or it was not possible to determine what was done). Thus, some publications (for different crops) were not used here because of the selection criteria. Seven papers could not be used. In some cases, corrections were made when enough information was available or the original data were available (in order to conduct a new analysis).

b Abbreviations: Bact. = bacteria, Phyto. = phytoplasma, Oomy. = oomycete, and Un. = unknown etiology.

${ }^{c}$ If $n$ was not constant across all observations, the mean $n(\bar{n})$ is reported (rounded to the nearest integer in the table). The range of $n$ or $\bar{n}$ is shown when BPL analyses were done for different sampling-unit sizes. 
Log base. Authors often report the intercept of the linear version of the BPL fitted to the data (see equations 10a to c). However, it is sometimes not clear whether natural (base $e$ ) or common (base 10) logs were used; the text may not agree with the presented graphs. As described above, this has no effect on the estimated $b$ (as long as the same log base was used on the left and right sides of the equation); however, if one is interested in knowing $A_{X}$ (equation 9a) as an example, one must know whether estimated $\log _{10}\left(A_{X}\right)$ or $\ln \left(A_{X}\right)$ is reported. Assuming that a graph is presented, then one often can figure out the $\log$ base (if $n$ is known and correctly reported). For instance, if there are estimated incidence values of approximately 0.5 , then the largest value of the binomial variance (count version)

TABLE 1

(Continued from previous page)

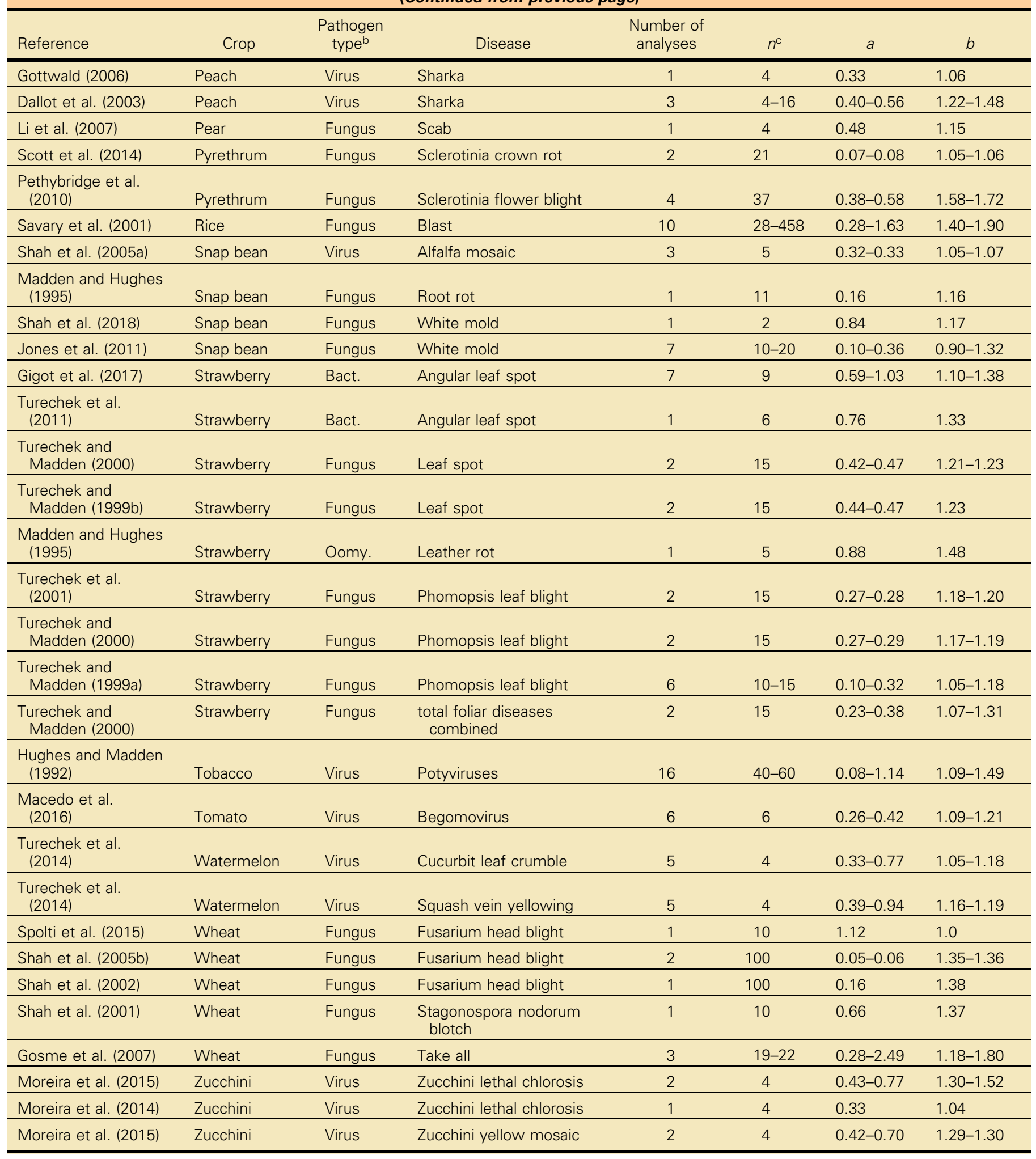


on the far right abscissa of the graph should be either $\log _{10}(n / 4)$ or $\ln$ $(n / 4)$. If the proportion version is used, the far right abscissa should be $\log _{10}(1 / 4 n)$ or $\ln (1 / 4 n)$. Likewise, if one knows the smallest $p$ used, then one can determine the smallest value of the transformed binomial variance that can occur on the far left of the graph using equation $9 \mathrm{a}$ or $9 \mathrm{~b}$ (a natural $\log$ is approximately 2.3 times the corresponding base-10 log). The ordinate of the log-log linear graph can also provide guidance on the $\log$ base used in calculation, based on the maximum variance that incidence can take (see later discussion on maximum variance).

Counts or proportions. It is not always clear whether the count (equations $9 \mathrm{a}$ and 10a) or proportion (equations $9 \mathrm{~b}$ and $10 \mathrm{~b}$ or $9 \mathrm{c}$ and 10c) model formulation is used. Descriptions in published methods sections may not be consistent with the graph or the analysis being conducted. It appears that, sometimes, authors reproduce the printed methods from a paper that utilized the count version of the BPL but actually use the proportion for their analysis, or vice versa. The estimate of $b$ is not affected by the choice of model formulation (with constant $n$, and assuming that the left- and righthand sides of the utilized model are consistent for counts or proportions), but the estimated intercept could be either $\ln \left(A_{X}\right)$ or $\ln \left(A_{p}\right)$ (assuming a natural log version here). As shown in equations $11 \mathrm{a}$ and $11 \mathrm{~b}$, the relationship between $A_{X}$ and $A_{p}$ is a nonlinear function of $n$ and $b$. The linear BPL graph (Figs. 1C, 2C, and 3) can also help here (assuming again that $n$ is known and reported). If natural logs are used, and there is at least one incidence value at or near 0.5 , then the largest value of the abscissa is either $\ln (n / 4)$ for counts or $\ln (1 / 4 n)$ for proportions.

Intercept transformation. Sometimes it is not specified whether the reported "intercept" is actually the intercept from the linear form of the model, such as $\ln \left(A_{X}\right)$, or whether the back-transformation has been made so that $A_{X}\left[=\exp \left(\ln \left(A_{X}\right)\right]\right.$ is directly reported (assuming the count version here with natural logs). The best way to figure this out is to generate a straight line with the reported results to see if they give the line shown in the BPL graph.

Synthesis of BPL parameter estimates. Using all the "tricks" in the previous section, we identified 230 useable regression analyses of observed data sets in the literature (Table 1). Results from theoretical simulations are not included here. Some papers evaluated different model-fitting methods on the same data, so we only preserved the results from one estimation method in our master database. Results from seven papers were not useable because there was not enough information given to determine what was done. From the listed "intercept" [e.g., $\ln \left(A_{p}\right), \ln \left(A_{X}\right)$ ], we estimated (approximated) $a, A_{X}$, and $A_{p}$ based on $n$ (or $\bar{n}$ for an analysis where the size of a sampling unit was not fixed) and estimated $b$ (equations $11 \mathrm{a}$ and $\mathrm{b}$ ).

Previously, Turechek et al. (2011) summarized the BPL results up to 2010. Some of the multiple results from a paper were generally reduced to a mean parameter-estimate result or result from a pooled analysis. We provide the 230 individual BPL results (after corrections based on the above comments) in Supplementary Table $\mathrm{S} 1$. Table 1 shows a range of results for each study and crop or disease. The estimate of $b$ ranged from 0.87 to 2.00 , with a mean of 1.24 and a median of 1.19 (Fig. 6). The frequency distribution was slightly right-skewed. In total, $75 \%$ of the estimates were $<1.33$ and $90 \%$ were $<1.51$. Only eight $b$ estimates were $<1$. Frequency distributions of estimated $a, A_{X}$, and $A_{p}$ were highly right-skewed; therefore, we present results for their natural-log transformations (Fig. 6). Even the transformed parameter estimates were somewhat right-skewed for $\ln \left(A_{p}\right)$ and $\ln \left(A_{X}\right)$. The goodness-of-fit of the BPL, as measured by $R^{2}$, was high for the 187 cases where this statistic was given. The median $R^{2}$ was 0.97 , with $75 \%$ of the values $>0.93$; $25 \%$ were $\geq 0.99$.

The value of $n$ (or $\bar{n}$ when the sampling-unit size was not fixed) used by the investigators ranged from 2 to 458 (the latter corresponding the aggregation of multiple contiguous quadrats), with a median of 10 (Fig. 6). In total, 75\% of $n$ values were less than 25 . Estimated $b$ increased in a reasonably linear manner with $\ln (n)$, $\ln$ $\left(A_{p}\right), \ln \left(A_{X}\right)$, and $\ln (a)$ (Fig. 6). In individual studies where more than one sampling-unit size was considered for the same dataset, $b$ generally increased directly with $n$ (Batista et al. 2008; Humeau et al. 2006). There was an especially strong relationship between $b$ and $\ln \left(A_{p}\right)$, in agreement with simulation results (Xu and Ridout 2000). The relationship between $b$ and $\ln (a)$ was stronger than found for the earlier subset of these results, as summarized by Turechek et al. (2011).

The coefficient of variation (CV) of estimated $b$ values across all studies was only $16 \%$, which we consider low, given the large diversity in cropping systems, pathogen types, environments, and sampling approaches (including $n$ ). However, this variation is reduced further when $b$ estimates where placed in four bins based on the quartiles of the frequency distribution of $n$. Then, CV was reduced to $11 \%$ for each of the first three $n$ categories and only increased to $16 \%$ for the largest $n$ category (corresponding to $n$ ranging from 26 to $>400$ ). Typically, there was even less variation when one looked at the estimates of $b$ from different datasets for a single pathosystem (with the same general sampling methods) (Gent et al. 2008; Turechek and Madden 1999a).

Estimated $\ln \left(A_{p}\right)$ and $\ln \left(A_{X}\right)$ were positively related to $\ln (n)$, although the relationship was considerably weaker for $\ln \left(A_{X}\right)$. There was a slight negative relationship between $\ln (n)$ and $\ln (a)$, which was also seen in Turechek et al. (2011). This is not surprising because $a$ decreases as a nonlinear function $n$ in equation 11 a (for values of $b>0$ with fixed $A_{p}$ and values of $b<2$ for fixed $A_{X}$ ).

\section{A GENERAL CONCEPTUALIZATION OF HETEROGENEITY OF DISEASE INCIDENCE}

A major advantage of the BPL is that one can use the model parameters to gain a general understanding of small-scale spatial pattern of disease incidence over the full range of incidence $(p)$ for a given system. This is done by comparing the variance and intracluster correlation $(\rho)$ for the BPL (with a given set of parameters) with corresponding values for selected other models for heterogeneity. These other models represent spatial randomness as well as the possible high and low extremes in heterogeneity for any given level of incidence. We demonstrate this using the results for the strawberry Phomopsis leaf blight data in Figure 3A (a subset of several datasets described by Turechek and Madden [1999a]). The fitted BPL line based on the count version of the BPL (equation 10a) is shown in Figure 7B, together with other lines and curves, including one for the standard binomial $\left[\ln \left(A_{X}\right)=0, b=1\right]$. For this example, the BPL $b$ parameter was estimated at 1.18 , which means that the observed variance is increasing faster than the binomial variance as $p$ increases toward $1 / 2$. In this example, the estimated $\ln (V)$ line crosses below the binomial line at $\ln [n p(1-p)]=-\ln \left(A_{X}\right) /(b-1)=-0.971 /$ $0.18=-5.4$, corresponding to $p$ of approximately 0.0003 (or $1-p$ of approximately 0.9997 ) with $n=15$. Based on an appraisal of the literature, it is not uncommon for the binomial and predicted BPL lines to cross at very low $p$ (and very high $1-p$ ), usually at a $p$ near or below the smallest value in the dataset. For the published BPL analyses described above, we calculated that the median crossover point was at $p=0.002 ; 75 \%$ of the crossovers were below $p=0.009$.

The corresponding intracluster correlations $(\rho)$ for the BPL and binomial lines are shown in Figure 7A for this example. (A similar graph is shown in Figure 9.5B of Madden et al. [2007]). As required, $\rho=0$ for the binomial (random) situation and, because $b>1$, $\rho$ changes systematically with $p$ for the BPL (equation 13), with a maximum of 0.168 at $p=1 / 2$. Careful appraisal of Figure 7A shows that $\rho$ falls below 0 at $p$ very close to 0 and 1 , at the same points where $\ln (V)$ crosses the $\ln [n p(1-p)]$ line in Figure 7B. Larger values of $\rho$ would require different combinations of $b$ and $A_{X}$. 
Maximum variance. There is an upper bound to the observed variance of disease incidence data. There is also a nonzero lower bound for the variance that can be considered. These bounds are useful for giving a perspective on the results of a BPL analysis. To see this, we return our attention to equation $6 \mathrm{a}$ (or $6 \mathrm{~b}$ ), which represents the observed variance at any specific value of $p$ (i.e., at a specific $\rho$ and $n$ ) (Collett 2003). As described above, equation 6a also satisfies the BPL when $\rho$ varies with $p$ according to equation 13 .
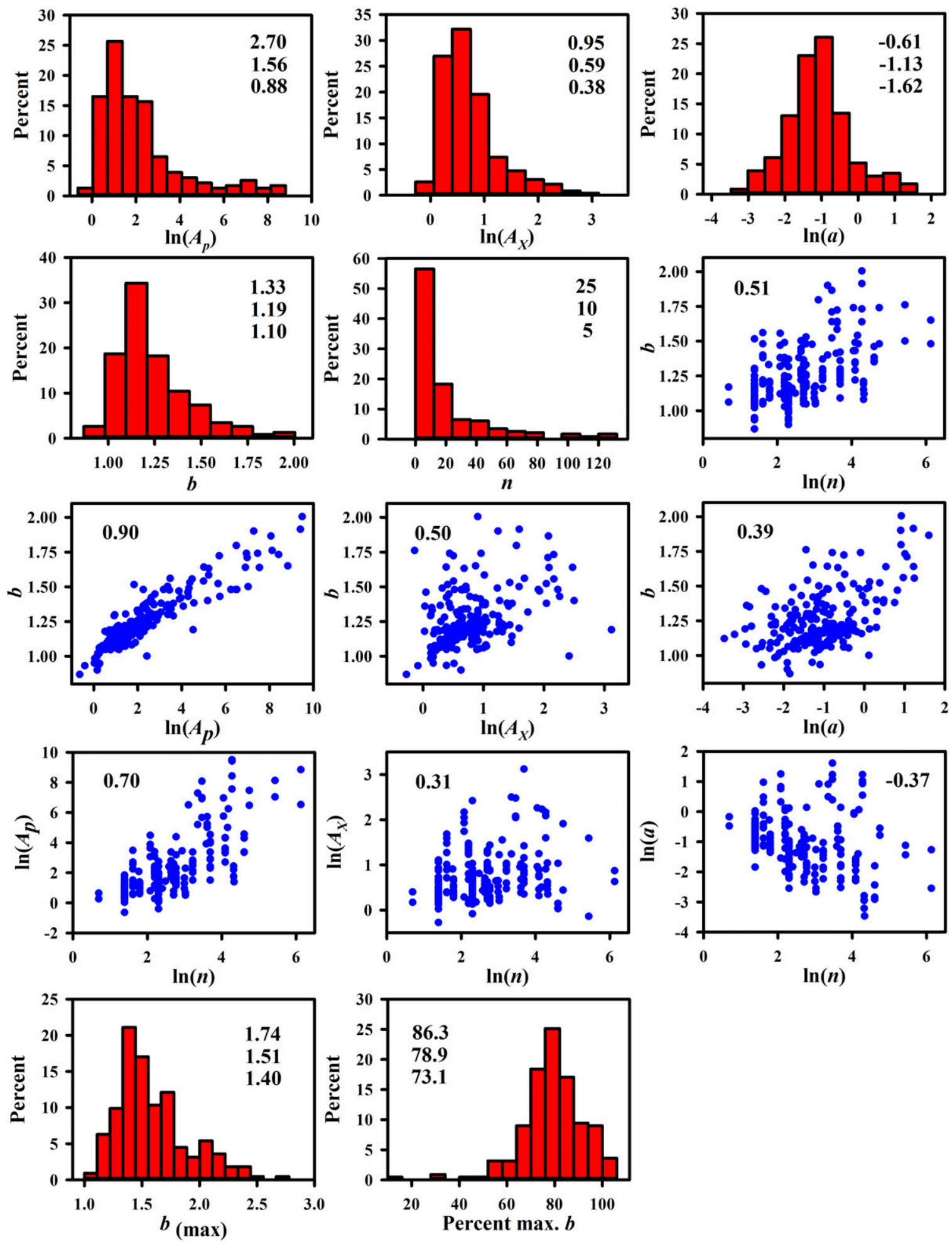

\section{FIGURE 6}

Empirical results of the binary power law (BPL) parameter estimates and sampling-unit sizes from the published literature. In any given paper, an estimate of only one of the following is given: $A_{x}, A_{p}$, or a (or its log transformation); we estimated the other two using equations $11 \mathrm{a}$ and $\mathrm{b}$ (using $n$ or $\bar{n}$ ). Last row: calculation (prediction) of the maximum possible $b\left(b_{(\max )}\right)$ for cases where estimated $b>1$, based on the published parameter estimates and $n$ or $\bar{n}$ (equation 14 , with assumptions in the text), and the closeness of estimated $b$ to estimated maximum ( $b_{(\% \text { max), }}$ percentage of the maximum). To better visualize the frequency distributions, some graphs are truncated (although all values are used in calculating statistics). The frequency distribution is truncated at 120 for $n$ and 3 for $b_{(\max )}$. Numbers within frequency graphs: three quartiles of the distributions. Number within scatter plots: rank correlation coefficient. 
We can now use this equation to see how $V$ varies with $p$ for different (constant or variable) $\rho$ values.

Using equation $6 a$ (or $6 b$ ) at a fixed $\rho$ is equivalent to using the BPL (equations 9a to c) with $b=1$ and $A_{X}=A_{p}=1+(n-1) \rho$. Using the maximum $\rho$ of 1 in equations $6 a$ and $b$, one finds that $1+(n-1) \times 1=n$ (the maximum value of $D)$, which leads to:

$$
\begin{aligned}
& V_{(\max )}=n V_{\text {bin }}=n[n p(1-p)]=n^{2} p(1-p) \\
& v_{(\max )}=n v_{\text {bin }}=n[p(1-p) / n]=p(1-p)
\end{aligned}
$$

As with the variance, in general, the largest value of maximum variance occurs at $p=1 / 2$; thus, that the largest maximum variance is given by $V_{(\max *)}=n^{2} / 4$ and $v_{(\max *)}=1 / 4$. For the Phomopsis leaf blight example, $\ln \left(V_{(\max )}\right)$ (equation $14 \mathrm{a}$ ) is plotted in Figure $7 \mathrm{~B}$ ("Max."); note that the maximum-variance line has the same slope

FIGURE 7

A, Intracluster correlation $(\rho)$ versus incidence $(p)$ and $\mathbf{B}$, log of the observed variance $[\ln (V]$ versus log of the binomial variance $[\ln (n p(1-p))]$ for several scenarios (count version of the binary power law [BPL]). The thick solid line represents the results for the Phomopsis leaf blight example in Figure 3A (where $\rho$ is determined from equation 13). Other lines represent the binomial (random) situation, different versions of the minimum $\rho$ or minimum $\ln (V$, as well as the maximum $\ln (V)$ (maximum $\rho$ is 1$)$. "Min.

$(\mathrm{BBD})$ " represents the minimum that is consistent with a $\beta$-binomial distribution, and "Min" represents the absolute minimum based on variances of correlated binary observations (Ridout et al. 1999). Equations are given in the text. as the binomial line (on the log-log linear graph) but an obviously different height. The largest value for this line is $\ln \left(15^{2} / 4\right)=4.03$ at the $\log$ binomial variance of $\ln [15 \times 0.5(1-0.5)]=\ln (15 / 4)=$ 1.322. We do not show the corresponding $\rho=1$ horizontal line on the intracluster correlation graph (Fig. 7A) because it is so much larger than the realized maximum from the BPL of approximately 0.16 .

A little algebra shows that the vertical distance between $\ln \left(V_{(\max )}\right)$ and $\ln \left(V_{\text {bin }}\right)$ is $\ln (n)$. This is $\ln (15) \approx 2.7$ in the example (i.e., the $\log$ of the largest possible $D[=n]$ ). (If we worked with the proportion version of the BPL, the vertical difference is also $\ln (n))$. Temporarily ignoring the fact that an observed variance, $V$, can be (slightly) less than the binomial variance (see below), we see that the BPL variance line primarily lies within the region defined by the binomial and maximum variance lines on the log-log scale (Fig. 7B). Therefore, one can visualize how far the BPL line is from the maximum at any level of the binomial variance (or, equivalently, at
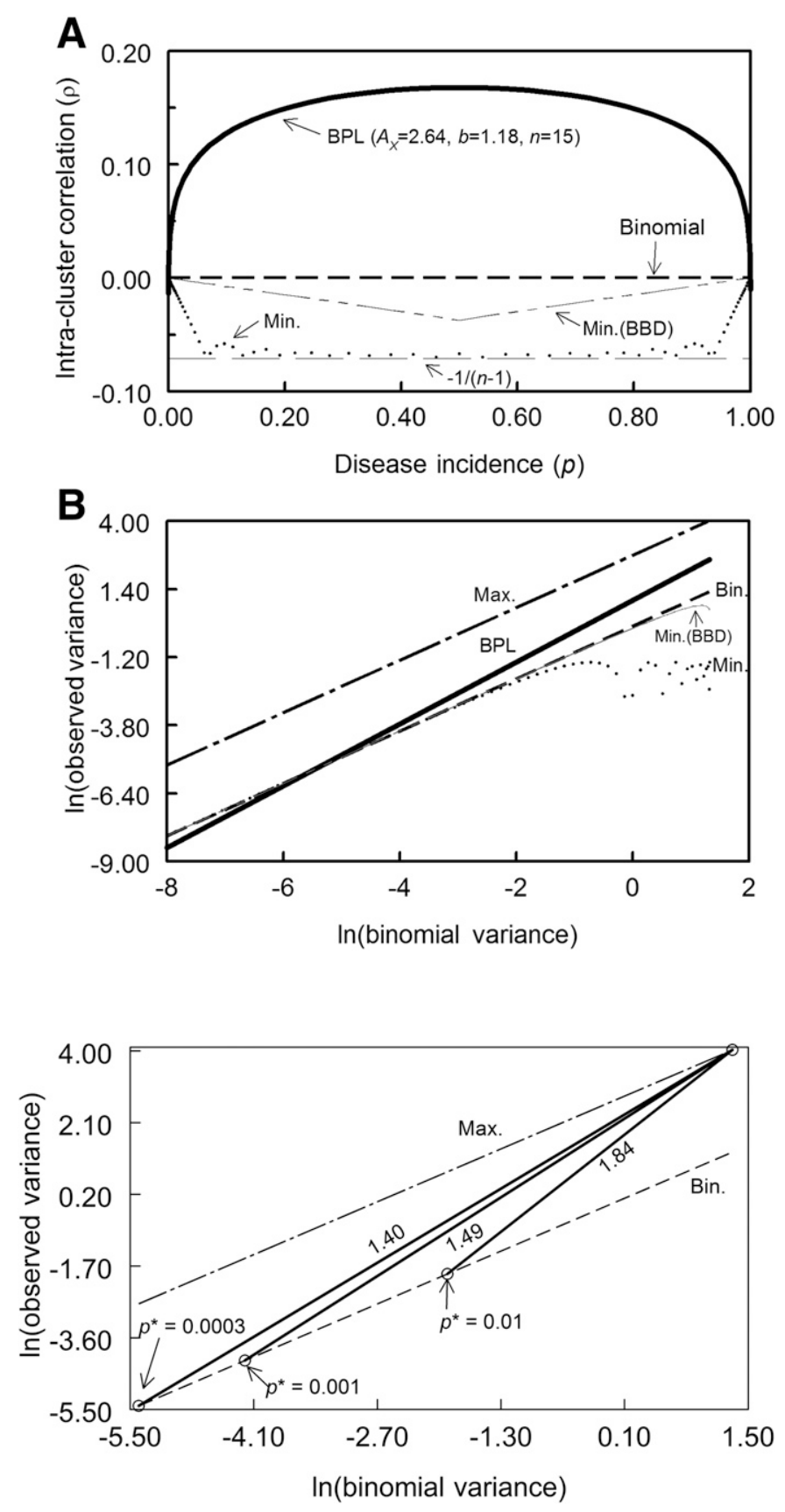

\section{FIGURE 8}

Relationship between log of the observed variance and log of the binomial variance for a conceptual example demonstrating the maximum $b$ parameter of the binary power law (BPL). "Max." and "Bin." represent the maximum and binomial variance lines, respectively (for

$n=15$ individuals per sampling unit). Solid lines represent three scenarios where the BPL lines cross the binomial line at three different

values of disease incidence $\left(p^{*}\right)$, and reach the maximum possible variance $\left(n^{2} / 4\right)$ at $p=1 / 2$. The case for $p^{*}=0.0003$ corresponds to the strawberry leaf blight example of Figure $3 \mathrm{~A}$. 
any level of $p$ ), and also see how rapidly the BPL line approaches the maximum (all on a log scale). This type of appraisal is not relevant for Taylor's power law because there is no definable upper limit to the variance for unbounded counts.

Minimum variance. Variances smaller than the binomial variance represent underdispersion, corresponding to regular or uniform patterns (Madden and Hughes 1995). We can use equation $6 \mathrm{a}$ or $6 \mathrm{~b}$ to determine the minimum possible variance of disease incidence $\left(V_{(\min )}\right.$ or $\left.v_{(\min )}\right)$ based on the minimum $\rho$ (at a given value of $p$ and $n$ ). The minimum possible $V$ is 0 for a random variable, which corresponds to the exact same value of $X$ in each sampling unit. $V=0$ in equation 6 a when $\rho=-1 /$ $(n-1)$; therefore, some references report $\rho_{\min }=-1 /(n-1)$. This would give $\rho_{\min }=-1 /(15-1)=-0.071$ for the strawberry Phomopsis leaf blight example; this lower limit is identified in Figure 7A. We cannot show the corresponding $\ln \left(V_{(\text {min })}\right)$ in Figure $7 \mathrm{~B}$ because $\ln (0)$ is undefined.

However, in general, it is not possible for discrete data to have the same value in each sampling unit (except as special cases). For instance, consider a hypothetical situation with $N=100$ sampling units observed, each with $n=10$ plants, and that $p=0.055$. This means that there would be a total of $55(=0.055 \times 100 \times 10)$ diseased plants across the 100 sampling units. It is obviously not possible for there to be $0.55(55 / 100)$ diseased plants in each sampling unit, which is the only way for the variance to be 0 . Thus, even with the most underdispersed situation, sampling units could not have the exact same number of diseased individuals, This means that, in general, the minimum variance must be $>0$ and $\rho_{\text {min }} \geq-1 /(n-1)$.

There is more than one possible $\rho_{\text {min }}$ to consider. Prentice (1986) and Smith (1983) showed that slightly underdispersed discrete data can be represented by the $\beta$-binomial distribution, a distribution that is usually used to characterize overdispersed data. Smith used the $\theta$ formulation of the $\beta$-binomial; therefore, we reparameterized this here as $\rho[=\theta /(1+\theta)]$ to be consistent with the rest of the presentation. When $X$ has a $\beta$-binomial distribution, the minimum $\rho$ is given by:

$$
\rho_{\min }=\max \left(\frac{-p}{n-p-1}, \frac{p-1}{n+p-2}\right)
$$

As shown in Figure 7A ("Min.(BBD)"), the minimum $\rho$ declines with increasing $p$, reaching a minimum of -0.037 (with $n=15$ ) at $p=1 / 2$, and then increases back to 0 . Relative to the binomial variance, this negative $\rho$ only has a slight effect on the minimum variance determined by using $\rho_{\min }$ in equation $6 \mathrm{a}$ (Fig. $7 \mathrm{~B}$ ):

$$
\mathrm{V}_{(\min )}=V_{\mathrm{bin}}\left[1+(n-1) \rho_{\text {min }}\right]=n p(1-\mathrm{p})\left[1+(n-1) \rho_{\text {min }}\right]
$$

The effect on the variance is mostly seen at incidence values between 0.4 and 0.6 (or $\ln \left(V_{\text {bin }}\right)$ values $>1.2$ [with $n=15$ ]). For most of the range of $p$, it is difficult to see the slight difference between the binomial- and the $\beta$-binomial-based minimum variance line. Although the $\beta$-binomial has been shown to describe a large number of observed plant disease distributions (Madden et al. 2007), we are not aware of efforts to explicitly describe underdispersed incidence data using the $\beta$-binomial.

It is possible for disease incidence to have a lower $\rho$ (and, hence, lower $V)$ than defined in equation $15 \mathrm{a}$, although the data would no longer have a $\beta$-binomial distribution. Based on standard formulas for the variances of correlated (binary) random variables (Prentice 1986; Ridout and Xu 2000; Ridout et al. 1999), the minimum $\rho$ for $X$ is given by:

$$
\rho_{\min }=\frac{-1}{n-1}+\frac{\omega(n, p)(1-\omega(n, p))}{n(n-1) p(1-p)}
$$

Where $\omega(n, p)=n p-\operatorname{INT}(n p)$ and $\operatorname{INT}(n p)$ is the integer (floor) function of the $n p$ expression. That is, INT( $\cdot)$ returns the integer portion of a real number; for example, $\operatorname{INT}(2.75)=2$. Equation $15 \mathrm{~b}$ does not describe a smooth relationship with $p$; rather, there can be multiple increases or decreases in the minimum $\rho$ as $p$ changes. We graph equation $15 \mathrm{~b}$ as individual points ("Min.") at specific $p$ values rather than as a line or curve in Figure 7A. Between $p$ of 0.2 and 0.8 , this minimum $\rho$ is close to or equal the absolute minimum of $-1 /(n-$ 1). Substituting equation $15 \mathrm{~b}$ for $\rho$ in equation $6 \mathrm{a}$, one can clearly see how much lower $\ln \left(V_{(\text {min })}\right)$ is compared with $\ln \left(V_{\text {bin }}\right)$ (Fig. 7B). The difference between the minimum and binomial variance is most pronounced in the example at incidence values between 0.05 and 0.95 , which translates to $\ln \left(V_{\text {bin }}\right)$ values greater than approximately -0.35 . At very small values of $p$ (for example, $p \leq 0.01$ ), $V_{(\min )}$ is almost as large as $V_{\text {bin }}$.

It is fairly common for the fitted BPL line to cross below the binomial line at a very low value of $p(1-p)$, which translates to $p$ very close to 0 and 1 (Figs. 1, 2, and 3). In the example in Figure 7B, this happens at approximately $p=0.0003$ (and $p=0.9997$ ). At such low values of $p$, the minimum variance is very similar to the binomial variance. Under these very-low- $p$ circumstances, therefore, the predicted BPL variance is slightly lower than the minimum possible variance. Although this is undesirable, it has little bearing on the interpretation of the BPL results for most of the range of incidence values (except possibly for development of sampling plans, as discussed below). For the example in Figures $3 \mathrm{~A}$ and $7 \mathrm{~A}$ and $\mathrm{B}$, there were no data points at or near this low crossover value of $p$, and the BPL provides a good fit over the observed range of incidences.

In our literature review, the estimated crossover point between the binomial and BPL lines was found to be, in general, near or (much) below the minimum visually observed $p$ in the dataset (for those datasets where we could make an appraisal). The large relative sampling error for very low incidence (Madden and Hughes 1999b) certainly contributes to uncertainty of the estimated variances at these extreme incidence values. As discussed by Perry and Woiwod (1992) and articles cited therein, there may be artifacts in measures of overdispersion at very low mean density values (such as when almost all of the sampling units are "empty" [free of the organism or disease of interest], with just one sampling unit occupied), which could influence power-law results; they discuss some expanded Taylor power law models to accommodate the possible artifacts. Gent et al. (2008) evaluated the influence of one type of low-incidence artifact, known as a singleton, on BPL results for powdery mildew of hops and found only a minor impact on parameter estimates. The possible behavior of the BPL at very low incidence values has also been addressed in theoretical studies, as will be summarized below.

A geometric argument. Given the importance of $b$ in reflecting the change in overdispersion with incidence (Fig. 7A), it is relevant to get a sense of the magnitude of an estimated $b$ compared with what is achievable for a particular sampling situation. We can use the binomial and maximum-variance lines (on the log-log linear graph) to provide guidance on the maximum $b$ that can occur for a given value of $n$. The calculation of this "maximum" assumes that the BPL (equations 9a to $\mathrm{c}$ or 10a to c) is an appropriate representation of the observed variances, the predicted BPL variance is larger than the minimum variance between a lower value of $p$ (denoted $\left.p^{*}\right)$ and upper value $\left(1-p^{*}\right)$, and that the BPL variance reaches $V_{(\max )}$ at $p=1 / 2$ (recalling that the highest possible $V_{(\max )}, V_{(\max )}$, occurs at $\left.p=1 / 2\right)$. Because the minimum and binomial variances are extremely close at very small $p^{*}$ (Fig. 7), we simplify the calculations here and assume that the binomial variance is, as an approximation, the minimum variance. Figure 8 can be used to visualize this calculation (for $n=15$ ). The binomial ("Bin.") and maximum possible variance ("Max.") lines are identical to those in Figure 7. The three solid lines are hypothetical BPL prediction lines that cross the binomial line at $p^{*}$ (and $1-p^{*}$ ), and reach $V_{(\max )}$ at $p=1 / 2$. (Note that the BPL variance below $p^{*}$ is not shown because it represents predictions lower than the minimum). If the lines cross at $p^{*}=0.0003$ (the result for the 
Phomopsis leaf blight example in Figures $3 \mathrm{~A}$ and 7), then one can see that the maximum possible $b$ is 1.40 (Fig. 8). The estimated $b$ is $84 \%\left(b_{(\% \max )}=100 \times 1.18 / 1.4\right)$ of the maximum possible value.

If the lines cross at $p^{*}=0.001$, then maximum $b$ is 1.49 ; if they cross at a much higher $p^{*}=0.01$, then maximum $b$ is 1.84 (Fig. 8). The higher the value of $p^{*}$ where the BPL line crosses above the binomial, the larger the maximum possible $b$. From a purely geometric argument, the larger the size of the sampling unit $(n)$, the greater the vertical distance between the binomial and maximum variance lines (Figs. 7 and 8), and the larger the region between the lines. With increasing $n, b$ can take on a wider range of values $>1$ and there is greater opportunity for "maximum" $b$ (at a given $p^{*}$ ) to be larger. It can be shown after a little algebra that the predicted maximum $b$ (when $n$ is a constant) is given by:

$$
b_{(\max )}=\frac{-\ln \left(\frac{n}{4 p *(1-p *)}\right)}{\ln (4 p *(1-p *))}
$$

for any selected $p^{*}$ [assuming that the BPL line for $\ln (V)$ equals $\ln$ $\left(V_{\left(\max ^{*}\right)}\right)=\ln \left(n^{2} / 4\right)$, its largest possible value, at $\left.p=1 / 2\right]$. When $n$ is variable, one could use $\bar{n}$ in equation 16 for an approximate relationship; there may be other ways of dealing with variable $n$ that have not been explored. In Figure $9, b_{(\max )}$ is presented for a range of $n$ and $p^{*}$ values. For instance, when the binomial and BPL lines cross at $p^{*}=0.001$, the maximum $b$ is $1.25,1.42,1.49,1.68$, and 1.81 at $n=4,10,15,40$, and 80, respectively.

It is interesting to note that the maximum $b$ values for $p^{*} \leq 0.001$ are all $<2$ for all the sampling unit sizes evaluated. Only at a (much) larger $p^{*}$ (e.g., $p^{*}=0.01$ in the graph) did the maximum $b$ exceed 2 at typical sampling unit sizes. Thus, it is not surprising from this geometric argument alone that (i) estimates of the BPL $b$ in the literature vary over a narrower range than found for Taylor's power law (Kendal 2004; Taylor et al. 1988) and (ii) estimated BPL $b$ values are positively correlated with $n$ (Fig. 6). Simulation modeling has provided a broader understanding of the range and magnitude of $b$ values that are achievable in epidemics (see below).

The above geometric arguments assume that the BPL variance reaches the highest possible $V_{(\max )}\left(V_{(\max *)}\right)$ at $p=1 / 2$ (Fig. 8). However, it is possible that an estimated $b$ could be larger than $b_{(\max )}$ if the analysis was done based on a narrow range of incidence values not close to $1 / 2$, the number of data points was small, the variances were imprecisely estimated before the regression analysis, or the analysis was done incorrectly (which cannot necessarily be judged by the publication). The estimate of $b$ (or the intercept) would be unreliable under these types of circumstances.

FIGURE 9

Maximum $b$ parameter of the binary power law (BPL) in relation to sampling-unit size $(n)$ and the point where the BPL line crosses the binomial line (label next to the line). This assumes that the BPL equals the maximum possible variance ( $n^{2} / 4$ for counts and $1 / 4$ for proportions) at $p=1 / 2$. Other assumptions are given in text.
We estimated $b_{(\max )}$ and $b_{(\% \max )}$ (based on the above assumptions) for the published BPL analyses when $b>1$ by first calculating the crossover point, $p^{*}$, from the estimates of $b$ and the intercept. Estimates of $b_{(\max )}$ ranged from 1.08 to 2.8 (Fig. 6, last row), not counting three outliers of 4.2, 4.2, and 11.0. The outliers came from regressions based on only five data points, with estimated $p^{*}>0.2$. Median $b_{(\max )}$ was 1.51 , and $75 \%$ of the maxima were $<1.74$. Median $b_{(\% \max )}$ was $79 \%$, with $75 \%$ of the values $<86 \%$ (Fig. 6 ). The $b_{(\% \max )}$ ranged from 10 to $107 \%$, which included eight values $>100 \%$. Six came from one study (Humeau et al. 2006), which was an unusual one because the plants were inoculated (most of the BPL analyses are done for naturally occurring epidemics). The table with their results apparently also had the intercepts and slope columns reversed; thus, there is some uncertainty about the parameter estimates. The other excessively large $b_{(\% \max )}$ was found with a very small number of data points, with very low disease incidence for all observations. Overall, it appears that the estimated $b$ values are fairly large relative to the maxima.

\section{MECHANISIMS}

Background on Taylor's power law. There have been major efforts over the years to explain, justify, predict, or even refute the appropriateness of Taylor's power law for representing the heterogeneity of discrete data with no upper bound. As stated by Giometto et al. (2015), the "widespread verification of TL [Taylor's law] has led many authors to suggest the existence of a universal mechanism for its emergence, although there is currently no consensus on what such a mechanism would be." Stochastic modeling, based on population dynamics or animal behavior, has been especially popular for this purpose (Anderson et al. 1982; Cohen 2014; Cohen et al. 2013; Perry 1988, 1994; Taylor and Taylor 1977; Taylor et al. 1983), and has resulted in some intense early arguments in the literature, as summarized by Kendal (2004). Taylor (1961) originally thought that $b$ represented a species characteristic but empirical observations and modeling showed that numerous physical and biological factors affect the magnitude of both $b$ and $\kappa$ (Downing 1986; Eisler et al. 2008; Kendal 2004).

Given that Taylor's power law describes the heterogeneity of biological, physical, and even numerical systems (Eisler et al. 2008; Kendal and Jørgensen 2011), it is unlikely that population-dynamic or behavioral processes alone could be sufficient to explain the ubiquity of the empirical law. With that in mind, there has been a new wave of theoretical studies aimed at explaining or understanding the ubiquity of Taylor's power law. Fronczak and Fronczak (2010) used the principles of statistical physics and the

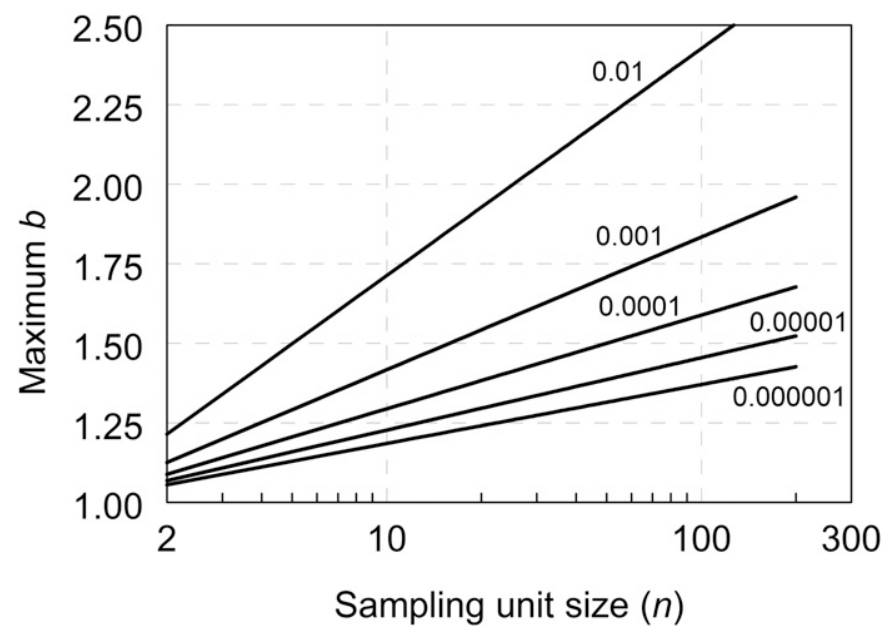


concepts of free energy and an external field to propose that Taylor's power law is the result of the second law of thermodynamics. Kendal and Jørgensen (2011) were skeptical of these arguments, stating "For systems known to exhibit Taylor's law, we found it difficult (if not impossible) to establish a biological correspondence with the postulated free energy and external physical field". Relying on a theory of exponential dispersion models and error distributions developed by Jørgensen (1997), Kendal (2004) showed that Taylor's power law results when the random variable has a so-called Tweedie dispersion model (a type of statistical distribution). This work is reminiscent of earlier research by Perry and Taylor (1985) on application of the Adès distribution to the Taylor power law.

Cohen and colleagues have continued to examine some of the diverse mechanisms generating Taylor's power law. Using various mathematical and stochastic methods, they have found that the variance-mean power-law relationship results from a wide range of stochastic processes, as well as from sampling in blocks from a skewed distribution (Cohen 2014; Cohen and Xu 2015; Cohen et al. 2013; Giometto et al. 2015).

BPL. Hughes and Madden (1992) and Madden and Hughes (1995) never postulated a mechanism generating the BPL. In fact, based on the experience with Taylor's power law, it is likely that there is no universal mechanism producing the BPL; multiple processes likely result in $V$ being a power function of $V_{\text {bin }}$ (equations 8 to 10). Even though the results from any single BPL analysis will not reveal a unique generating mechanism, there is interest in moving our understanding of the BPL forward, to better interpret parameter values in relation to possible biotic and abiotic processes.

It is well established that overdispersion-characterized by $V>$ $V_{\text {bin }}$ or $D>1$ (equation 5) - often occurs when data are collected using cluster sampling (Hughes et al. 1996), due to either the spatial correlation of disease status of individual plant units within sampling
FIGURE 10

Estimated $b$ and $A_{p}$ parameters of the binary power law (BPL) fitted to the output of a stochastic simulator (Xu and Ridout 2000) for a range of epidemic and sampling conditions: $\mathbf{A}, b$ versus sampling-unit size ( $n$, number of plants in a sampling unit); $\mathbf{B}, b$ versus $A_{p}$ (determined after back-transforming the estimated intercept of equation $10 \mathrm{~b})$; and $\mathbf{C}, A_{p}$ versus $n$. Results are for epidemics starting with four infected plants randomly located in the simulated field (subset of the results for different starting conditions and sampling-unit sizes in Xu and Ridout 2000, Figure 1)

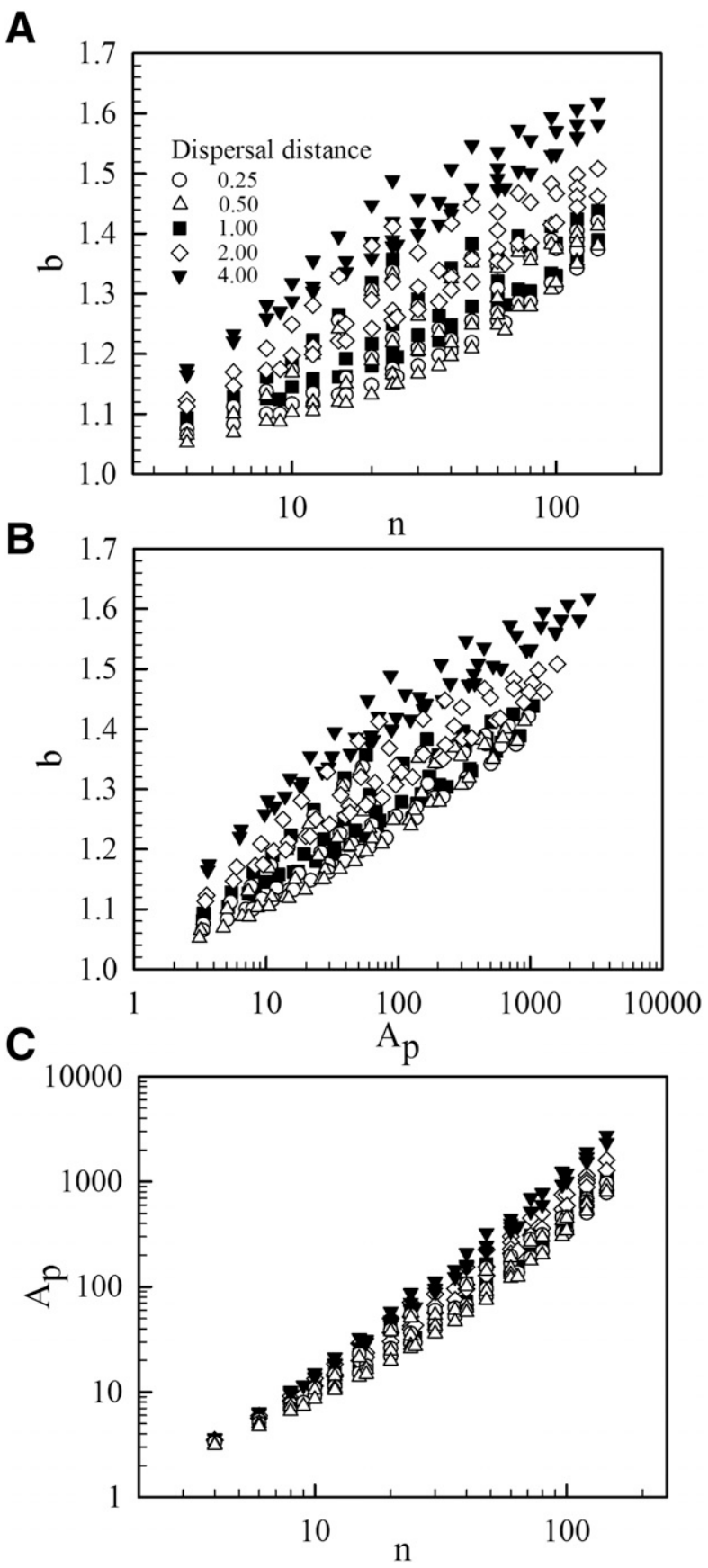


units or the random variation of the (latent) probability of disease across the clusters (sampling units) (Collett 2003; Madden and Hughes 1995; Madden et al. 2007; Ridout and Xu 2000). It is not immediately obvious that $V$ should vary systematically with $V_{\text {bin }}$ as a power function (other models are conceivable for $V$ and $p$ before looking at the data), or that the exponent of the power function is mostly between 1 and 2. Compared with investigations of Taylor's power law, there have only been a relatively small number of theoretical studies evaluating the BPL, although these studies all confirm, in general, that the BPL (with $b \geq 1$ ) is a natural consequence of stochastic population-dynamic processes for disease incidence (Gosme and Lucas 2009; Shah and Bergstrom 2002; Turechek et al. 2011; Xu and Madden 2014; Xu and Ridout 2000).

$\mathrm{Xu}$ and Ridout (2000) used a previously developed stochastic, spatially explicit epidemic simulation model (Xu and Ridout 1998) to evaluate the effect of a range of epidemiological and sampling factors on BPL goodness-of-fit and on the magnitude of the BPL parameters. Sporulation rate, latent period, infectious period, and median dispersal distance (for a Cauchy contact distribution) are specified in the model. For the current purposes, the spatial lattice of plants was partitioned into quadrats of different lengths and widths, and the variance and mean incidence across quadrats for each of the sampling times throughout the epidemics were analyzed with equation 10b. The BPL provided a good fit to the variances for virtually all of the simulated epidemics; estimated $b$ and $\ln \left(A_{p}\right)$ were mostly $>1$, with $b$ ranging from 0.93 to 1.94 . For a given set of initial epidemic conditions, both BPL parameters increased with: increasing median length of the dispersal function (contact distribution), the latter being a measure of the distance that spores can move from where they are produced to where they can cause infections; and the size of the sampling units $(n)$ (see Figure 10 for one set of initial conditions). The relation between $b$ and $n$ was also found for the field datasets (Fig. 6), although with higher variability in the bivariate relation for field studies, presumably because of there was no control for other factors (dispersal and so on) in the graphs in Figure 6. Effects of $n$ on the BPL parameters are not surprising because these parameters characterize the $\rho$ (equations 7 and 13) and $D$ (equations 5 and 12) overdispersion metrics, and it is well known that sampling-unit size is a major determinate of overdispersion in general (Madden and Hughes 1995; Madden et al. 1995b; Navas-Cortés et al. 2008; Ridout and Xu 2000). In fact, $\rho$ will often decline with increasing $n$ (at a given $p$ ) because there is opportunity for more variation in disease status of individuals within sampling units-with corresponding lower variation in counts or proportions among sampling units-as $n$ becomes larger.

Later work with the same $\mathrm{Xu}$ and Ridout (2000) simulator showed that $b=1$ primarily for small sampling-unit size and very short dispersal distance (Turechek et al. 2011). Interestingly, Shi et al. (2016) recently showed with a different simulator that dispersal distance also affects Taylor's power law $b$ parameter.

Perhaps surprisingly, Xu and Ridout $(1998,2000)$ also found that initial conditions such as the initial number of diseased plants and their large-scale pattern in the spatial lattice (uniform, random, or clumped) at time 0 had substantial effects on the BPL parameters for the entire epidemics. For instance, mean estimated $b$ (averaged over the "other" factors) increased from 1.17 to 1.25 as initial number of diseased plants increased from 1 to 9 ; mean $b$ was $1.12,1.22$, and 1.28 for clumped, regular, and random initial (large-scale) spatial patterns, respectively. There were similar results for estimated ln $\left(A_{p}\right)$. There were also substantial interaction effects of initial conditions and size of the sampling units on the BPL parameter estimates.

Xu and Madden (2014) used a generic stochastic spatial-contact model, "without appealing to any particular biological mechanism", to explore the extent to which the BPL must hold for representing the heterogeneity of counts with an upper bound. One specifies the number (and distance) of individual spatial neighbors (neighborhood size) that affect infection of an individual at a given site at a given time and the strength of the neighborhood influence on infection. The spatial unit was the individual (e.g., a plant), and then the units were combined into quadrats (sampling units) of different sizes for BPL analysis. The BPL (equation 10b) was found to provide a very good fit to the variance data (with $b \geq 1$, in general) whenever there was a positive influence of the neighbors on infection (translated as a positive correlation in the disease status of first or higher-order neighbors [primarily] within quadrats, or when there was no neighborhood influence within quadrats [the homogeneous case]). The only time when the usual BPL (equation 10b) did not provide a satisfactory fit for Xu and Madden (2014) was when neighbors had a negative effect on infection at a site (translated as a negative spatial correlation), such that an infection in a neighbor decreased the probability of disease at a given site.

In general, there is positive or zero spatial autocorrelation for observed plant disease data from field investigations (Madden et al. 2007), with little evidence of negative correlation. Theoretical models also generally lead to positive correlations (Filipe and Gibson 2001). Thus, the simulation results in $\mathrm{Xu}$ and Madden (2014) give one possible reason why it is so common to find a good fit of the BPL in plant pathology. Ridout and Xu (2000) described how the spatial correlation structure of the disease status of individuals (e.g., plants) within sampling units affects the variance of counts among sampling units $(V, v)$ and the corresponding intracluster correlation $(\rho)$.

In the theoretical work by $\mathrm{Xu}$ and Madden (2014), mean estimated $b$ (at a given sampling unit size) increased monotonically with increasing neighborhood influence or increasing magnitude of spatial autocorrelation of neighbors; estimated $b$ equaled 1 , in general, for the zero-correlation (homogeneity, binomial) situation. The intercept, $\ln \left(A_{p}\right)$, was highly and positively correlated with $b$. A resampling investigation of the simulation results (where a small fraction of the total population of sampling units was repeatedly sampled with replacement) showed that, except for the homogenous $(b=1)$ case, estimated $b$ and $\ln \left(A_{p}\right)$ varied for any single set of simulation conditions. Thus, giving an exact meaning to small differences in estimated BPL parameters (e.g., $b=1.3$ versus 1.2 ) is challenging, and probably impossible.

Gosme and Lucas (2009) made an interesting theoretical discovery using their spatially nonexplicit simulation model, which appears to be most suitable for a nearest-neighbor type of transmission process. They found a biphasic or multiphasic (split line) power-law relationship for the observed and binomial variances. In particular, they found a steep linear relation between $\ln (v)$ and $\ln \left(v_{\text {bin }}\right)$ at very low disease incidence, followed by a less steep linear relation between $\ln (v)$ and $\ln \left(v_{\text {bin }}\right)$ (with a $b$ relatively close to 1) over most of the range of $p$. The break point occurred at an incidence of approximately 0.001 to 0.01 ; with empirical datasets, there are typically few variance observations for such low incidence values. At high $p$, there could also be other phases (line segments) in the log-log graph (with more subtle changes in $b$ ). These simulation-based results are reminiscent of the earlier stochastic population-dynamic findings of Anderson et al. (1982) with Taylor's power law, who showed that $b$ can vary theoretically with the mean density of organisms.

The findings by Gosme and Lucas (2009) prompted Turechek et al. (2011) to evaluate the published BPL results up through 2010. They could find no evidence of a bi- or multiphasic relationship in published graphs, even when very low incidence values were included in the analysis, such as with the BPL results by Gottwald et al. (2007) (Fig. 1). Our review of the literature up through early 2017 did not reveal any additional evidence for the split-line relation. Using the spatially explicit mechanistic simulator of $\mathrm{Xu}$ and Ridout (1998), Turechek et al. (2011) evaluated factors that might lead to a split-line phenomenon. They did find that, in the extreme, the biphasic (split-line) relationship could indeed occur; 
however, this was most apparent only with very short median dispersal distances (consistent with nearest-neighbor spread) and large $n$. An example of the phenomenon is in Figure 11. The initial break point was found to be between incidence values of 0.001 and 0.01 , in general. Very large sampling units probably are not suitable to characterize spatial patterns when the dispersal distance is very short.

The limited theoretical studies conducted to date certainly support the ubiquity of the BPL for describing the heterogeneity of disease incidence or other random count variables with an upper bound of $n$. However, a good fit of the BPL to observed variance data does not tell you anything about a particular mechanism generating the data, because several different epidemiological processes as well as sampling and starting conditions could be responsible for the resulting $b$ and $A_{p}$ (or $A_{X}$ or $a$ ) parameter estimates. Cohen (2014) made similar points about Taylor's power law when he stated that it "is impossible to infer the underlying stochastic process solely from the observation that a random variable satisfies TL [Taylor's law]". Nevertheless, these theoretical studies show that a given sampling-unit size, dispersal, and initial conditions are major factors affecting parameter values.

Uncertainty about an underlying cause for the BPL, however, does not interfere with its use for characterizing overdispersion over a wide range of disease incidence values in order to better understand spatiotemporal dynamics of epidemics. BPL parameters can also be of direct value in several applications.

\section{APPLICATIONS}

We consider here three general applications of the BPL: sampling, data analysis of treatment effects, and prediction in a hierarchy.

Sample size. The most common application of the BPL is in sampling for disease incidence. Broadly, the objective of sampling can be either to:

1. estimate disease incidence $(\hat{p}=\bar{x})$ with a desired (minimum) level of precision, defined in terms of the half-width of the confidence interval for incidence or the coefficient of variation of the mean $[(C=s e(\hat{p}) / \hat{p})$, where $s e(\hat{p})$ is the estimated standard error $]$; or

2. test a hypothesis about the level of incidence (i.e., classify incidence as being above or below a threshold that could be of relevance for a control intervention).

FIGURE 11

Split-line binary power law (BPL) example based on stochastic simulation results of Xu and Ridout (2000). Sampling unit size of $n=64$ $(=8 \times 8)$ with a half-Cauchy dispersal distance of 1 . Proportion version of BPL is used; solid line corresponds to binomial situation. First break point occurs at $\ln [p(1-p) / 64] \approx-9$, which corresponds to $p \approx 0.007$.

Vertical broken lines correspond to $p=0.001$ and 0.01 . Details and additional results given in Turechek et al. (2011).
The BPL can be applied to both of these general objectives. Readers should see chapters 9 and 10 in Madden et al. (2007) and Madden and Hughes (1999b) for details on these topics; we only give a brief synopsis of using the BPL to determine the required number of sampling units $(N)$.

The estimated standard error (se) of incidence for a given sampling unit size $(n)$ can be written as:

$$
s e(\hat{p})=\sqrt{\hat{a}[\hat{p}(1-\hat{p})]^{\hat{b}} / N}
$$

where $a$ can be obtained from equation 11a and b (Madden and Hughes 1999b). The fact that the BPL parameters are estimates adds a level of uncertainty to the se estimate (increasing the mean square error of the estimated $s e$ ) but it is typical to simply use equation 17 and assume that the BPL parameters are known constants. The half-width of the confidence interval and also $C$ depend on the se. We focus here only on controlling the magnitude of $C$. We can divide both sides of equation 17 by the estimated $p$ in order to obtain the coefficient of variation of the mean, and then solve for $N$. The resulting equation is:

$$
N=\hat{a} \hat{p}^{\hat{b}-2}(1-\hat{p})^{\hat{b}} C^{-2}
$$

An example sample-size graph is shown in Figure 12 based on the parameter estimates for the pyrethrum flower blight example in Figure 3F (Pethybridge et al. 2010). To estimate mean incidence with $C=0.20(20 \%)$, for instance, one requires $N=36$ sampling units (of $n=37$ each) when incidence is 0.05 but only 5 sampling units when incidence is 0.50 . Much higher numbers are needed for $C=0.10$.

Several papers have used the BPL to determine $N$ (Gent et al. 2007a,b, 2008; Hughes and Gottwald 1999; Shah et al. 2005a; Turechek and Madden 1999b; Turechek et al. 2001). A challenge with this calculation is that one needs at least a rough prediction of $p$ to use equation 18 . This is not necessarily too difficult of a burden, because one would "know" (from previous experience) that $p$ is low early in an epidemic and higher later. This may be sufficient for preparing sample-size guidelines.

In some situations, disease incidence can only be determined after the sampling is completed (e.g., through use of polymerase

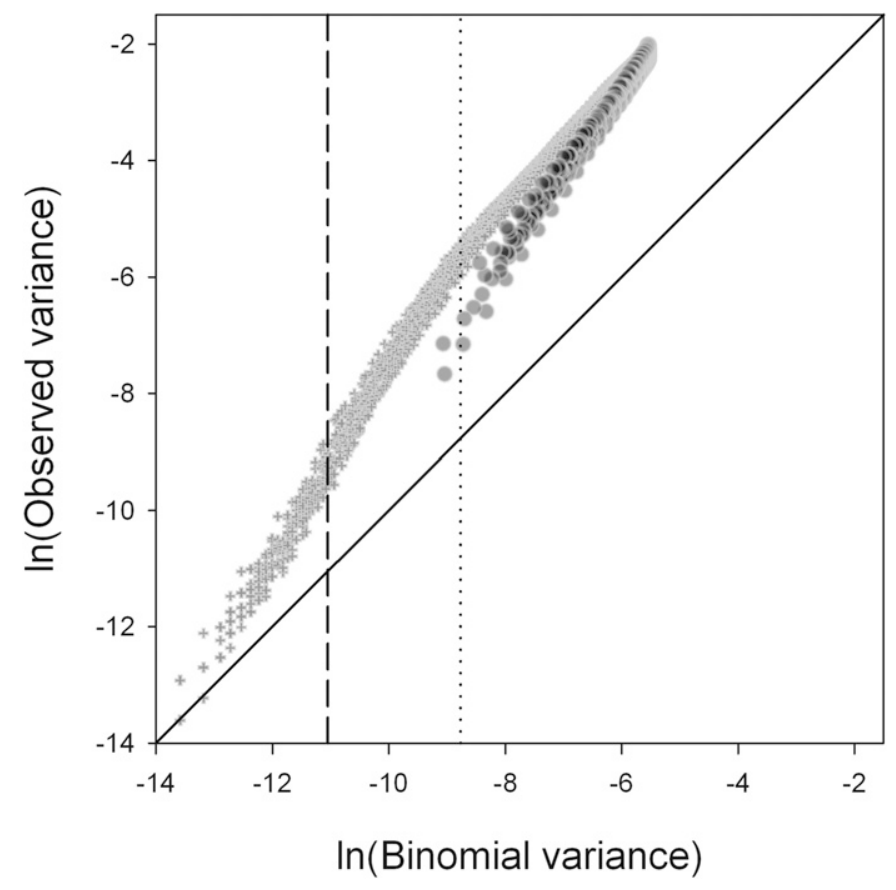


chain reaction assays in the lab). However, when assessment is based on visible symptoms during the sampling bout, sequential sampling methods can be used in the field. That is, one collects data until the total number of diseased individuals at a given sample size $\left(T_{N}=\Sigma X=p n N\right)$ exceeds a threshold. Determining the $T_{N}$ versus $N$ curve at a given $C$ or a given half-width of the confidence interval is somewhat complicated with the BPL (when $b>1$ ), requiring an iterative solution based on the estimates of $a$ and $b$. In particular, one first calculates

$$
\tau_{N}=C^{2} \hat{a}^{-1} n^{2 \hat{b}^{-2}} N^{2 \hat{b}^{-1}}
$$

for values of $N$ from 1 to a large value, and then iteratively determines the value of $T_{N}$ at each $N$ that satisfies:

$$
\tau_{N}=T_{N}^{\hat{b}-2}\left(n N-T_{N}\right)^{\hat{b}}
$$

Details are given by Madden et al. (1996) and Madden and Hughes (1999b, pages 1,095 to 1,096 ). Several papers have developed sequential sampling plans based on BPL results (Carisse et al. 2009; Gent et al. 2007a,b; Jones et al. 2011; Turechek and Madden 1999b; Turechek et al. 2001). In general, required $N$ increases with increasing overdispersion (as defined by the combination of $b$ and $a$ ).

The BPL can be used to determine the required number of sampling units to make a decision about the level of disease incidence; that is, to classify incidence as being below or above a threshold or test a hypothesis about incidence being below or above a constant. Madden and Hughes (1999b) developed a sequential sampling approach based on the so-called sequential probability ratio test (SPRT). Their approach was inspired by the pioneering in-depth sampling research by economic entomologists for making decisions on arthropod density (Binns et al. 2000). The advantage of this sequential-sampling approach is that it naturally allows for different levels of precision of the estimated $p$, depending on the distance from the threshold (hypothesized) value. For instance, if a disease management decision depends on whether or not mean incidence exceeds 0.2 , then one does not need to have a precisely estimated $p$ (i.e., a small $N$ ) if the estimated incidence is 0.8 or 0.02 but one needs a very precise estimate (i.e., large $N$ ) if estimated incidence is 0.18 or 0.22 .

Readers are referred to section 11.5 in Madden et al. (2007) and pages 1,096 to 1,101 in Madden and Hughes (1999b) for details on applying the BPL to this form of sequential sampling. Several papers have utilized the SPRT-based sampling methods for disease incidence based on BPL results (Carisse et al. 2009; Gent et al. 2007a,b; Jones et al. 2011; Turechek et al. 2001).

All of these sampling methods assume that $a$ and $b$ are known constants (for a given $n$ ), although the parameter estimates obviously vary for a given pathosystem (Table 1). Nevertheless, these methods work well even when $a$ and $b$ are only known as approximations for the new data being collected in the sample.
Although not covered here, there are several approaches to confirm whether the sampling objective is met, such as a desired CV being achieved or a correct decision being made (Madden and Hughes 1999b; Turechek et al. 2001). Some of the tools involve bootstrapping and simulation to determine the operating characteristic curve and the average sample number curve. Economic entomologists have confirmed that using previously determined Taylor power law parameters works well for achieving sampling objectives (Binns et al. 2000).

Data transformation. By definition, discrete data have variances that are functions of the means (Stroup 2013). This creates a dilemma with traditional analysis of variance (ANOVA) for assessing effects of treatments or other factors on counts or proportions, because an assumption of ANOVA is that the variance is independent of the mean (Schabenberger and Pierce 2002). As discussed in the introduction, some of the early work with Taylor's power law and its predecessors was concerned with using the $b$ parameter to transform the counts so that the new variable had an approximately constant variance (and approximately symmetrical distribution) at all means (Bartlett 1936; Bliss 1941; Perry 1987). With unbounded counts described by Taylor's power law, one can use the transformation $Y=g(X)=X^{\varphi}$, where $\varphi=1-b / 2$, to stabilize variances; this transformation is tightly linked with the more general and popular Box-Cox variancestabilizing transformation (Schabenberger and Pierce 2002).

The Box-Cox or Taylor-based transformation is not appropriate for proportions such as disease incidence (Piepho 2003). If $X$ has a binomial distribution (i.e., $A_{p}=A_{X}=1, b=1$ ), then the angular transformation of the proportion $x$ can be used to approximately stabilize the variances (except for $p$ very close to 0 or 1 ):

$$
y=g(x)=\sin ^{-1}(\sqrt{x})
$$

where $\sin ^{-1}(\cdot)$ is the arcsine function and, as always in this article, $x(=X / n)$ represents proportions. The transformation can also be applied directly to estimated probability of disease, $\hat{p}=\bar{x}[=(\Sigma x) / N]$, for each level of the factors in the experiment. More complex functions have been proposed that do a better job of stabilizing variances over the full range of binomial proportions, especially for proportions very close to 0 and 1, and for producing a symmetrical distribution (Schabenberger and Pierce 2002; Yu 2009). When the BPL $b$ parameter is not equal to 1 , the variance (or the degree of overdispersion) changes in a nonlinear manner with the mean proportion (equation 10a to c). Thus, the angular transformation is not optimum for stabilizing proportion data, in general.

As far as we are aware, no analytical function $g(x)$ has been derived to stabilize the variances for a variable described by the BPL, except when $b=1$ (angular transformation) or when $b=2$ (logit). In their original BPL paper, Hughes and Madden (1992) took a numerical ad hoc approach to develop a polynomial function that can approximately stabilize variances of disease incidence. The empirical transformation is directly proportional to $b$. Due to the
FIGURE 12

Predicted number of sampling units needed to obtain a coefficient of variation of the mean $(C)$ equal to 0.10 or 0.20 in relation to disease incidence $(p)$. Curves based on equation 18 and the binary power law results for the pyrethrum flower blight example in Figure 3F (Pethybridge et al. 2010).

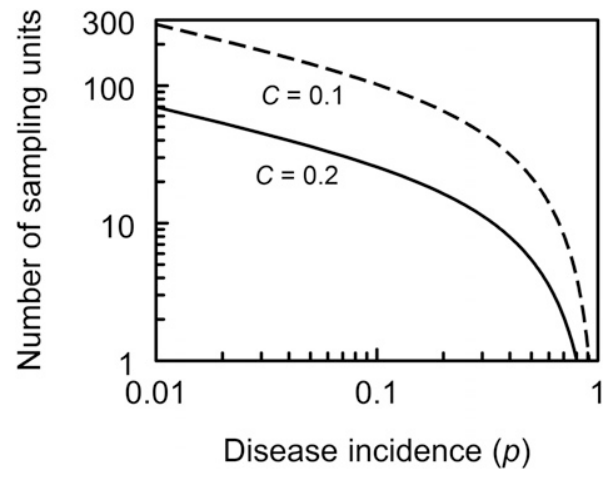


complexity of the numerically derived $g(x)$, it is not surprising that it has seldom been used since its introduction.

Piepho (2003) developed a different transformation to stabilize variances and produce symmetric distributions (close to normality) for proportion variables. The new folded exponential transformation is more general than the angular one (equation 19) because it involves a parameter $(\phi)$, estimated from the data, that depends on the degree of overdispersion and skewness of the data. The function can be written as $y=g(x)=\{\exp (\phi x)-\exp [\phi(1-x)]\} / 2 \phi$ for all nonzero values of $\phi$. The variance-stabilizing property of the transformation suggests that $\phi$ is performing the same function as $b$ of the BPL (in terms of producing variance stability). However, we have not found any direct links between these two parameters (unpublished); any relationship would only be numerical and approximate, at best. We think researchers should seriously consider the folded exponential transformation (Piepho 2003) when they are conducting analyses of disease incidence that require a stable variance.

Generalized linear mixed models. There have been growing arguments against the use of transformations to meet the statistical requirements of ANOVA or linear (i.e., normal) mixed models in recent years (Stroup 2013; Warton and Hui 2011). Transformations force the (altered) data to conform to a statistical model (including the distributional assumptions of the model); a better approach is to choose a model that conforms to the data. Analysis based on a generalized linear model (GLM) (for fixed effects and data with a distribution in the exponential family [Poisson, negative binomial, binomial, gamma, beta, and so on]) or generalized linear mixed model (GLMM) (for random and fixed effects with exponentialfamily distributions) allows the investigator to match the model (and its assumptions) to the data. Gbur et al. (2012) and Stroup (2013) give extensive coverage of this topic and discuss the many advantages of GLM and GLMM for analyzing discrete data (such as unbounded counts or counts with an upper bound).

In an early paper, Hughes and Madden (1995) presented a wide range of GLM-based methods to analyze (overdispersed) disease incidence data in designed experiments. Among other things, they covered methods suitable for nonconstant overdispersion, as found with data conforming to the BPL. Their work was based on fixedeffects-only (i.e., GLM) models. Piepho (1999) showed how some of the models in Hughes and Madden (1995) could be expanded to include random effects, allowing for the analysis of data from a much wider range of experimental designs. Madden et al. (2002) further evaluated these GLMM for several disease-incidence data sets obtained from designed experiments. They also presented an ad-hoc quasi-likelihood method to account for nonconstant overdispersion (as described by $b>1$ with the BPL) but found no clearcut improvement compared with the other GLMM being considered.

\begin{tabular}{|c|c|c|c|c|c|c|}
\hline \multicolumn{7}{|c|}{ 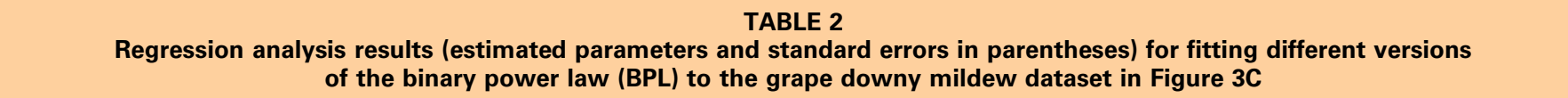 } \\
\hline M1 & $10 a$ & $\ln (\hat{V})$ & OLS & $\ln \left(A_{x}\right)$ & $0.56(0.111)$ & $1.512(0.133)$ \\
\hline $\mathrm{M} 2$ & $10 b, 22$ & $\ln (\hat{v})$ & OLS & $\ln \left(A_{p}\right)$ & $2.78(0.762)$ & $1.447(0.140)$ \\
\hline M5 & $9 b$ & $\hat{v}$ & NL (gamma) & $\ln \left(A_{p}\right)$ & $2.73(0.717)$ & $1.421(0.132)$ \\
\hline M6 & $10 \mathrm{~b}$ & $\ln (\hat{v})$ & Robust (MM) & $\ln \left(A_{p}\right)$ & $2.80(0.800)$ & $1.449(0.147)$ \\
\hline M7 & $10 \mathrm{~b}$ & $\ln (\hat{v})$ & RMA & $\ln \left(A_{p}\right)$ & $3.34(0.647)^{d}$ & $1.551(0.119)^{\mathrm{c}}$ \\
\hline \multicolumn{7}{|c|}{$\begin{array}{l}\text { a Ordinary least squares (OLS) fit for models } \mathrm{M} 1 \text { to } \mathrm{M} 3 \text { will give the same results if } n \text { is constant ( } n \text { is not constant here). } \\
\text { b } N L=\text { non-linear and } \mathrm{MM}=\text { modified maximum-likelihood type. } \\
\text { c General form of equation } 23 \mathrm{~b} \text {, with }-b \ln (n) \text { replaced by } \mathrm{cln}(n) \text {, where } c \text { is a parameter; } \hat{c}=0.133(0.784 \text { ). With this extra parameter, equation } 23 \mathrm{~b} \\
\text { is no longer the BPL of equations } 10 \mathrm{a} \text { to c. } \\
\text { d Standard error based on bootstrap sampling for reduced major axis (RMA) regression. }\end{array}$} \\
\hline
\end{tabular}

\section{FIGURE 13}

Relationship between disease incidence at the upper scale (proportion of sampling units with one or more diseased individual) and incidence at the lower scale (proportion of hops leaves with powdery mildew).

Data are from the "row" dataset of Turechek and Mahaffee (2004).

Predictions of higher-level incidence are based on the binomial distribution (equation 20a), $\beta$-binomial distribution, or effective sample size

(equation 20c), where the $v$ parameter in the latter is determined based on the binary power law results and equation 21 . The latter two curves overlap in this specific example.

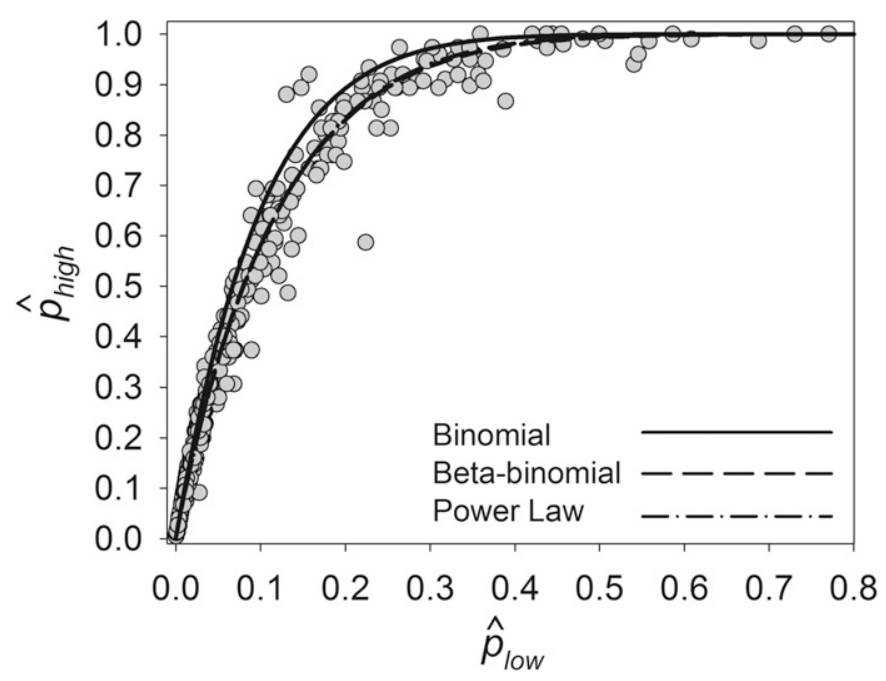


Park and Park (2008) take a related but more complex approach to the overdispersion problem.

Predictions in a hierarchy. Knowledge of the statistical distribution of disease incidence can be exploited for prediction. One special case involves predictions of disease in a spatial hierarchy (Gottwald 2006; Hughes and Gottwald 1999; Hughes et al. 1997a; Li et al. 2007; Shah et al. 2005a; Turechek and Madden 2001; Turechek and Mahaffee 2004; Turechek and McRoberts 2013; Xu and Madden 2002). For instance, one can use $p$ and the zero-term of the statistical distribution of $X$ [the probability that $X=0, \operatorname{Prob}(X=0)]$ to determine the probability that a sampling unit has one or more diseased individuals (i.e., that the sampling unit is occupied). If the sampling unit is a plant and the leaves on each plant were assessed for disease, then (mean) leaf disease incidence can be used to predict the probability that a plant is diseased. To see this, we will temporarily use $p_{\text {low }}$ instead of $p$ for the probability of an individual being diseased at the lowest level in the hierarchy. If $X$ has a binomial distribution, the probability of no diseased individuals in a sampling unit is given by $\operatorname{Prob}(X=0)=\left(1-p_{\text {low }}\right)^{n}$. The probability of one or more diseased individuals in a sampling unit, designated as $p_{\text {high }}$, is then given by:

$$
p_{\text {high }}=1-\left(1-p_{\text {low }}\right)^{n}
$$

The distribution of disease incidence usually is not binomial, however, but a distribution with a higher variance than the binomial, such as the $\beta$-binomial (Hughes and Madden 1993; Madden and Hughes 1995). With a $\beta$-binomial distribution, $p_{\text {high }}$ is given by:

$$
p_{\text {high }}=1-\left(1-p_{\text {low }}\right)^{E}
$$

where $E$ is complex summation function involving $p_{\text {low }}$ and the $\beta$-binomial $\theta$ parameter $[=\rho /(1-\rho)]$, as given by equation 11 in Madden and Hughes (1999a). Although use of equation 20b works fine in theoretical studies, it is much more practical for data analysis, and for intuitive insight, if the exponent is a constant for any single value of $p_{\text {low }}$. To this end, Madden and Hughes (1999a) showed that $p_{\text {high }}$ for a $\beta$-binomial distribution can be very accurately predicted using a model of the form of equation 20a, with $n$ replaced with a so-called effective sample size, v:

$$
p_{\text {high }}=1-\left(1-p_{\text {low }}\right)^{v}
$$

The $v$ parameter depends on $n$ and $\rho$ (or $\theta$ ). In general $v \leq n$; the higher the degree of overdispersion, the smaller $v$ is relative to $n$. $v$ can be approximated with an inverse polynomial function of $n$ and $\theta$ (Madden and Hughes 1999a):

$$
v \approx n / c_{0}+c_{1} \theta+c_{2} n+c_{3} \theta n+c_{4} \theta^{2}+c_{5} \theta^{2} n
$$

where $c_{0}=0.98135, c_{1}=0.8179, c_{2}=0.004958, c_{3}=0.30387$, $c_{4}=-0.3471$, and $c_{5}=-0.08475$. When the BPL describes the heterogeneity of incidence, $\rho$ can be predicted from $a$ and $b$ (equation 13), and $\theta$ calculated from $\rho$ (or $\theta$ determined directly from $a$ and $b$ ). Then, $v$ is estimated at each value of $p_{\text {low }}$ using equation 21 and, finally, equation $20 \mathrm{c}$ is used to predict the probability of disease at the higher scale.

An example relationship between $p_{\text {high }}$ and $p_{\text {low }}$ is given in Figure 13. Note that direct curve-fitting of the $p_{\text {high }}: p_{\text {low }}$ relationship is not needed; the two-level relationship for incidence is described strictly based on characterization of the heterogeneity at the lower level. One could also use curve fitting as an alternative way of describing the relationship (Carisse et al. 2011; Turechek and Madden 2003; Turechek and Mahaffee 2004). Whenever there is overdispersion (i.e., small-scale disease aggregation), $p_{\text {high }}$ at a given $p_{\text {low }}$ is lower than when the data are represented by a binomial distribution (i.e., for a random or homogeneous process). Hughes et al. (1997a) further showed, using disease progress models, that overdispersion at the lower level in the hierarchy leads to reduction in the rate of disease increase at the higher level. Turechek and Madden (2003) expanded on the approach in Madden and Hughes (1999a) to consider several additional models for the two-level problem as well as for a more complex three-level hierarchy (e.g., leaflets, leaves, and plants; or leaves, stems, and plants). Additional discussion and methods are found in Turechek and McRoberts (2013) and Shah et al. (2018).

The relationship in equations $20 \mathrm{a}$ or c can be turned around, so that one relates $p_{\text {low }}$ to $p_{\text {high }}$. This falls under the heading of group sampling, an approach that is very efficient for estimating $p_{\text {low }}$ from an estimate of $p_{\text {high }}$ (Hughes et al. 1997a; Madden et al. 2007, section 10.8).

\section{MODEL FITTING}

The BPL can be fitted to data using various techniques based on either nonlinear equations $9 \mathrm{a}$ to $\mathrm{c}$ or the linear equations $10 \mathrm{a}$ to $\mathrm{c}$. There is no a priori best model-fitting method, although most researchers have not explored the implications of different methods on the BPL parameter estimates.

With actual data, one typically bases the BPL analysis on the logtransformed estimated observed variances (equations $2 \mathrm{a}$ and $\mathrm{b}$ ) and $\log$-transformed estimated binomial variances (equations $4 \mathrm{a}$ and $\mathrm{b}$ ), the latter being a function of $\hat{p}$. Theory shows that $\hat{V}$ (or $\hat{v}$ ) has a gamma distribution (Schabenberger and Pierce 2002), which is (highly) right-skewed unless the number of samples $(N)$ is extremely large. In addition to the skewness feature, the variance of a gammadistributed random variable increases with the expected value of the distribution. The distribution of a log-transformed estimated variance (or any right-skewed gamma distributed variable) typically has much less skewness and may be (roughly) symmetrical, even though the transformation does not strictly give a normal random variable.

The statistical version of equation $10 \mathrm{~b}$ for the proportion version of the BPL can be written as:

$$
\ln (\hat{v})_{j}=\ln \left(A_{p}\right)+b \ln [\hat{p}(1-\hat{p}) / n]_{j}+\varepsilon_{j}
$$

where the $j$ subscript refers to a specific observation $(j=1, \ldots, T$, with $T$ representing the total number of variance observations in the dataset), $\ln [\hat{p}(1-\hat{p}) / n]$ is the predictor variable, and $\varepsilon$ is the random error (residual). Almost all published papers have used ordinary least squares (OLS) linear regression to fit the $\log -\log$ based model to the data (Table 1). The usual assumption for OLS is that $\varepsilon$ in equation 22 has a normal distribution with constant variance (Schabenberger and Pierce 2002), although linear least squares is robust to moderate departures from these assumptions. Given the effect of the log transformation on skewed distributions, analysis based on the $\log -\log$ equations $10 \mathrm{a}$ to $\mathrm{c}$ thus seems reasonable.

When the size of the sampling units $(n)$ is fixed, one obtains the exact same parameter estimates using the statistical versions of equations $10 \mathrm{a}, \mathrm{b}$, or c. One simply uses equations $11 \mathrm{a}$ and $\mathrm{b}$ to convert between the different "intercept" terms. However, if $n$ varies among the $T$ variance values in the BPL analysis, then the three statistical models do not give equivalent results. This is demonstrated with the grape downy mildew example shown in Figure 3C, when $n$ ranged from 10 to 17 among the $T=18$ points, with a mean $n$ of 12.4. The estimated $b$ was 1.512 for the count version (statistical version of equation 10a, model M1 in Table 2), 1.447 for the 
proportion version (equation $10 \mathrm{~b}$ or 22 , model M2), and 1.509 for the " $p(1-p)$ " proportion version (equation 10c, model M3). Obviously, the results are quite similar but they are not identical.

One can better understand why nonconstant $n$ leads to different results by looking at algebraically equivalent versions of equations $10 \mathrm{a}$ and $\mathrm{b}$ :

$$
\begin{gathered}
\ln (V)=\ln \left(A_{X}\right)+b \ln (n)+b \ln [p(1-p)] \\
\ln (v)=\ln (A p)-b \ln (n)+b \ln [p(1-p)]
\end{gathered}
$$

When $n$ is fixed, only the sum of the first two terms on the righthand side [e.g., $\left.\ln \left(A_{p}\right)-b \ln (n)\right]$ can be estimated as a single constant (intercept), not the two individual constants. However, when $n$ is variable, the BPL can be seen as a model with two variables, $\ln [p(1-p)]$ and $\ln (n)$, with one parameter $(b)$ for the sum of the two variables. One can see that $\ln (V)$ increases with $b \ln (n)$ but that $\ln (v)$ decreases with $b \ln$ $(n)$. Of course, independent of the BPL, $\ln (v)$ is negatively related to $2 \ln$ $(n)$, because $\ln (v)=\ln (V)-2 \ln (n)$, by definition; thus, it is difficult to see the net result of variable $n$ when fitting the models. In our view, there is no clear best model-formulation choice at this time for variable $n$, and future research will need to investigate the implications of variable $n$ on BPL analysis.

One approach is to generalize the BPL to include a separate parameter for the $\ln (n)$ term $c$, so that $b \ln (n)$ is replaced by $c \ln (n)$ in equation 23b. Fitting the statistical version of this model to the grape downy mildew example (proportion version) resulted in $\hat{b}=1.506$ and $\hat{c}=0.137$ (Table 2, model M4). Note that this more general model is not the BPL; $c$ cannot be identified for datasets with constant $n$.

One might be tempted to fit the BPL by using nonlinear least squares with an assumed (constant-variance) normal distribution for the residuals $(\varepsilon)$. For instance, the statistical version of equation $9 \mathrm{~b}$ could be written as:

$$
\hat{v}_{j}=A_{p}[\hat{p}(1-\hat{p}) / n]_{j}^{b}+\varepsilon_{j}
$$

However, we recommend against this approach because of the positive skewness of the distribution of the response variable $(\hat{v})$. Instead, one could explicitly use the gamma distribution for $\hat{v}$, utilizing either nonlinear or generalized linear modeling methods (Perry and Woiwod 1992; Schabenberger and Pierce 2002, section 6.7.7). With a gamma distribution for $\hat{v}$, the estimate of $b(1.421$, model M5) was almost the same as found when assuming a homogeneous normal distribution for $\ln (\hat{v})$ (1.446, model M2) (Table 2). In some analyses of other datasets (unpublished), we found similar estimates of $b$ in our comparisons of models M5 and M2, which lends support to the usual approach for fitting the BPL with OLS linear regression methods.

OLS may suffer when there are extreme observations of the response variable (outliers) or extreme observations of the predictor variable (highleverage points). This may happen, for instance, when most of the incidence values are approximately $50 \%$ (at or near the maximum binomial variance), and there are relatively few very low or high incidence values (where observed variances are small, by definition). This situation can be seen, to some extent, in the Phomopsis cane and leaf spot of grape example in Figure 3D (Nita et al. 2012). Many of the variance observations are clustered near the upper right corner of the graph. With these situations, robust regression methods, which are less sensitive to the usual distribution assumptions, may be desirable (Schabenberger and Pierce 2002). Nita et al. (2012) fitted the BPL to the data in Figure 3D using both OLS and the so-called modified maximumlikelihood-type (MM) robust regression methods (Yohai 1987), and obtained almost the same parameter estimates. In fact, the two fitted lines are actually shown in Figure 3D, which overlap almost completely over the range of incidence values because of the very similar parameter estimates. Previously, Madden et al. (1995a) compared robust regression (different from the MM method used here) with OLS regression for fitting the BPL to several grape downy mildew data sets. For the example in Figure 3C, MM robust regression (equation 22) resulted in an estimated $b$ of 1.449 (Table 2, model M6), very similar to that found with model M2. Although we did not see an advantage of robust regression in this case, the method should be seriously considered whenever there are large outliers or high-leverage points coupled with a small number of variance observations.

Standard linear and nonlinear regression methods, including robust methods, assume that the predictor (independent) variable is not subject to variability or that it is measured without error (Sokal and Rohlf 1995). Obviously, the estimated binomial variance, $\ln [\hat{p}(1-\hat{p}) / n]$, is determined with error, due to sampling and disease-assessment inaccuracy. Error in the predictor results in a biased $b$ parameter estimate (compared with the parameter for the underlying true relationship), with the estimate being smaller than the true slope value. If one knew the error in the predictor across all the data sets in the regression analysis, one could use error-invariable parameter estimation methods to fit the BPL to data (Seber and Wild 2003). In the typical situation where the error is not known, one can use reduced major axis (RMA) regression or major axis regression to fit the model (Smith 2009; Sokal and Rohlf 1995). RMA is equivalent to geometric mean regression, which can be appropriate when the error in the response and predictor are of equal magnitude, as well as when it is not clear which of the two variables should be the response variable or the predictor variable. RMA has previously been used by some for Taylor's power law (Taylor and Woiwod 1982), although almost all investigators use OLS. Madden et al. (1995a,b) previously compared the geometric mean and OLS parameter estimates in early papers using the BPL.

For the grape example here (Fig. 3C), the estimate of $b$ was 1.551 when RMA was used to fit the proportion version of the BPL (Table 2, model M7), which is higher (as required) than found for model M2. The difference in slopes was very small here; this difference would increase as the variability in the $v: v_{b i n}$ relationship increased. Both the OLS and RMA fitted lines are shown in Figure $3 \mathrm{C}$ but one can barely see the two different lines at the largest values of the binomial variance. RMA and related so-called "orthogonal" regression methods have remained controversial in statistics (Smith 2009), and we do not find that these methods are needed, given the high $R^{2}$ typically found for BPL analyses. Moreover, it seems natural to us to consider $\ln (v)$ [or $\ln (V)$ ] as the actual response variable.

\section{CONCLUDING REMARKS}

Referring to the BPL two decades ago, Hughes et al. (1997b) stated "Only further investigation of real patterns of disease incidence will determine how widely observed the characteristics of equations $(3.3 \mathrm{a}, \mathrm{b}, \mathrm{c})$ will turn out to be." Their equations $3.3 \mathrm{a}$ to $\mathrm{c}$ correspond to our equations $9 \mathrm{c}$ and $\mathrm{d}$ and $8 \mathrm{~b}$. The results summarized here (Figs. 1, 2, 3, and 6; Table 1) and in Turechek et al. (2011) certainly support the generality of the BPL for representing the spatial heterogeneity (overdispersion) of observed disease incidence data. A small number of studies from the ecological literature for nondisease proportion data provide further support for the BPL. Simulation studies have demonstrated that the BPL is consistent with known stochastic epidemiological processes, and is generally appropriate whenever there is zero or positive spatial correlation of the disease status of the neighboring plants composing the sampling units (Xu and Madden 2014; Xu and Ridout 2000). The BPL can be used together with other spatial methods for small-scale (e.g., index of dispersion and overdispersed discrete distributions) and larger-scale (e.g., spatial autocorrelation, semivariograms, SADIE, and cross-product statistics) patterns in order to develop a more comprehensive understanding of the spatial processes in botanical epidemics (Li et al. 2012; Madden et al. 2007). 
Although researchers often focus on means (or other location parameters such as medians) in their experiments or surveys, there is growing awareness that variances also are important summary parameters to be considered (Kriss et al. 2012; Nita et al. 2008). For instance, an experimental factor may affect both the mean and variance in a systematic way, the variance being a measure of the degree of heterogeneity or overdispersion of discrete data. In addition to being of interest to epidemiologists and other population scientists, this has practical importance because the magnitude of variances affects the estimates of the means with GLM and GLMM (Gbur et al. 2012). Research in plant pathology has clearly shown that disease incidence is generally overdispersed (Madden et al. 2007), and the BPL results have shown that the overdispersion (represented by $\rho$ or $D$ ) typically changes systematically with disease incidence. Above, we discussed some of the ways of dealing with overdispersion in sampling, data analysis, and prediction in a hierarchy using the BPL. This is not an all-inclusive list of applications.

So that their reported BPL results can be reasonably interpreted, researchers should always specify the sampling-unit size $(n), \log$ base being used, whether the analysis was based on the proportion or count version of the model, and whether the reported "intercept" is on the log scale or back-transformed. Furthermore, the range of incidence values in the regression analysis should be reported as well as the standard errors of the estimated parameters. There are several valid model fitting methods, although the commonly used OLS regression analysis on log-transformed variables is reasonable. Compared with the results for Taylor's power law (Taylor et al. 1988), the estimated $b$ parameters of the BPL in the literature appear to be less variable among studies or datasets, with smaller upper values, showing a slower rate of increase in the observed variance with increase in the random-distribution variance (on a log-log scale). The upper bound for the variance of disease incidence (Fig. 7) clearly attenuates the possible values of $b$ (Figs. 8 and 9); therefore, it is recommended that researchers determine in future studies how far their estimates of $b$ are from their maximum $\left(b_{(\max )}\right)$ values for a given $n$ and $p^{*}$. Simulations show that, although biotic and abiotic factors affect the BPL parameters, a small number of sampling units used to determine variances can lead to imprecision in the parameter estimates.

In conclusion, the BPL has been a catalyst for a deeper appreciation of the wider heterogeneity properties of disease incidence in plant populations and how we can use these properties in epidemic analysis. Although the BPL certainly leads to a very useful graph for succinctly representing heterogeneity over the full range of incidence values, its major value may have been in leading to advances in sampling for incidence, data analysis, predictions in a spatial hierarchy, and theoretical modeling of epidemics, all of which can increase our understanding of epidemics. The theory and applications were not necessarily foreseen when the original brief article was published a quarter of a century ago.

\section{ACKNOWLEDGMENTS}

We thank T. Gottwald, S. Pethybridge, D. Gent, and E. Del Ponte for sharing data with us.

\section{LITERATURE CITED}

Anderson, R., Gordon, D., Crawley, M., and Hassell, M. 1982. Variability in the abundance of animal and plant species. Nature 296:245-248.

Arnold, K. L., Golino, D., and McRoberts, N. 2017. A synoptic analysis of the temporal and spatial grapevine leafroll disease in an historic Napa vineyard and experimental vine blocks. Phytopathology 107:418-426.

Bartlett, M. S. 1936. The square root transformation in analysis of variance. Suppl. J. R. Stat. Soc. 3:68-78.

Bassanezi, R. B., Bergamin Filho, A., Amorim, L., Gimenes-Fernandes, N., Gottwald, T. R., and Bové, J. M. 2003. Spatial and temporal analyses of citrus sudden death as a tool to generate hypotheses concerning its etiology. Phytopathology 93:502-512.

Batista, L., Bassanezi, R. B., and Laranjeira, F. F. 2008. Comparative epidemiology of citrus tristeza in Cuba and citrus sudden death in Brazil. Trop. Plant Pathol. 33:348-355.

Benson, D. M., Grand, L. F., Vernia, C. S., and Gottwald, T. R. 2006. Temporal and spatial epidemiology of Phytophthora root rot in Fraser fir plantations. Plant Dis. 90:1171-1180.

Biggs, A. R., Turechek, W. W., and Gottwald, T. R. 2008. Analysis of fire blight shoot infection epidemics on apple. Plant Dis. 92:1349-1356.

Binns, M. R., Nyrop, J. P., and van der Werf, W. 2000. Sampling and Monitoring in Crop Protection. CABI Publishing, Oxon, UK.

Bliss, C. I. 1941. Statistical problems in estimating populations of Japanese beetle larvae. J. Econ. Entomol. 34:221-232.

Bonnot, F., De Franqueville, H., and Lourenço, E. 2010. Spatial and spatiotemporal pattern analysis of coconut lethal yellowing in Mozambique. Phytopathology 100:300-312.

Campbell, C. L., and Madden, L. V. 1990. Introduction to Plant Disease Epidemiology. John Wiley \& Sons, New York.

Carisse, O., Meloche, C., Boivin, G., and Jobin, T. 2009. Action thresholds for summer fungicide sprays and sequential classification of apple scab incidence. Plant Dis. 93:490-498.

Carisse, O., Meloche, C., and Turechek, W. W. 2011. Spatial heterogeneity, incidence-incidence and incidence-lesion density relationship of apple scab (Venturia inaequalis) in managed orchards. Eur. J. Plant Pathol. 130:349-365.

Cochran, W. G. 1977. Sampling Techniques, 3rd ed. John Wiley \& Sons, New York.

Cohen, J. E. 2014. Stochastic population dynamics in a Markovian environment implies Taylor's power law of fluctuation scaling. Theor. Popul. Biol. 93:30-37.

Cohen, J. E., and Xu, M. 2015. Random sampling of skewed distributions implies Taylor's power law of fluctuation scaling. Proc. Natl. Acad. Sci. USA 112:7749-7754

Cohen, J. E., Xu, M., and Schuster, W. S. F. 2013. Stochastic multiplicative population growth predicts and interprets Taylor's power law of fluctuation scaling. Proc. R. Soc. B 280:20122955.

Collett, D. 2003. Modelling Binary Data, 2nd ed. Chapman \& Hall/CRC, Boca Raton, FL.

Dallot, S., Gottwald, T., Labonne, G., and Quiot, J. B. 2003. Spatial pattern analysis of sharka disease (Plum pox virus strain M) in peach orchards of southern France. Phytopathology 93:1543-1552.

Downing, J. A. 1986. Spatial heterogeneity: Evolved behaviour or mathematical artefact? Nature 323:255-257.

Dutilleul, P. R. L. 2011. Spatio-Temporal Heterogeneity: Concepts and Analyses. Cambridge University Press, Cambridge, UK.

Eisler, Z., Bartos, I., and Kertész, J. 2008. Fluctuating scaling in complex systems: Taylor's law and beyond. Adv. Phys. 57:89-142.

Filipe, J. A. N., and Gibson, G. J. 2001. Comparing approximations to spatiotemporal models for epidemics with local spread. Bull. Math. Biol. 63: 603-624.

Fisher, R. A. 1925. Statistical Methods for Research Workers. Hafner Publishing, New York.

Fronczak, A., and Fronczak, P. 2010. Origins of Taylor's power law for fluctuation scaling in complex systems. Phys. Rev. E 81:066112.

Gbur, E. E., Stroup, W. W., McCarter, K. S., Durham, S., Young, L. J., Christman, M., West, M., and Kramer, M. 2012. Analysis of Generalized Linear Mixed Models in the Agricultural and Natural Resources Sciences. American Society of Agronomy, Madison, WI.

Gent, D. H., Fransworth, J. L., and Johnson, D. A. 2012. Spatial analysis and incidence-density relationships for downy mildew on hop. Plant Pathol. 61:37-47.

Gent, D. H., Mahaffee, W. F., and Turechek, W. W. 2006. Spatial heterogeneity of the incidence of powdery mildew on hop cones. Plant Dis. 90:1433-1440.

Gent, D. H., Turechek, W. W., and Mahaffee, W. F. 2007a. Sequential sampling for estimation and classification of the incidence of hop powdery mildew I: Leaf sampling. Plant Dis. 91:1002-1012.

Gent, D. H., Turechek, W. W., and Mahaffee, W. F. 2007b. Sequential sampling for estimation and classification of the incidence of hop powdery mildew II: Cone sampling. Plant Dis. 91:1013-1020.

Gent, D. H., Turechek, W. W., and Mahaffee, W. F. 2008. Spatial and temporal stability of the estimated parameters of the binary power law. Phytopathology 98:1107-1117.

Gigot, C., Turechek, W. W., and McRoberts, N. 2017. Analysis of the spatial pattern of strawberry angular leaf spot in California nursery production. Phytopathology 107:1243-1255.

Gilligan, C. A. 1982. Statistical analysis of the spatial pattern of Botrytis fabae on Vica faba: A methodological study. Trans. Br. Mycol. Soc. 79:193-200.

Giometto, A., Formentin, M., Rinaldo, A., Cohen, J. E., and Maritan, A. 2015. Sample and population exponents of generalized Taylor's law. Proc. Natl. Acad. Sci. USA 112:7755-7760. 
Gosme, M., and Lucas, P. 2009. Cascade: An epidemiological model to simulate disease spread and aggregation across multiple scales in a spatial hierarchy. Phytopathology 99:823-832.

Gosme, M., Willocquet, L., and Lucas, P. 2007. Size, shape and intensity of aggregation of take-all disease during natural epidemics in second wheat crops. Plant Pathol. 56:87-96.

Gottwald, T. 2006. Epidemiology of sharka disease in North America. EPPO Bull. 36:279-286.

Gottwald, T. R., Bassanezi, R. B., Amorim, L., and Bergamin Filho, A. 2007. Spatial pattern analysis of citrus canker-infected plantings in São Paulo, Brazil, and augmentation of infection elicited by the Asian leafminer. Phytopathology 97:674-683.

Guan, Q., Chen, J., Wei, Z., Wang, Y., Shiyomi, M., and Yang, Y. 2016. Analyzing the spatial heterogeneity of number of plant individuals in grassland community by using power law model. Ecol. Modell. 320: 316-321.

Hughes, G. 1990. Characterizing crop responses to patchy pathogen attack. Plant Pathol. 39:2-4.

Hughes, G., and Gottwald, T. R. 1999. Survey methods for assessment of citrus tristeza virus incidence when Toxoptera citricida is the predominant vector. Phytopathology 89:487-494.

Hughes, G., and Madden, L. V. 1992. Aggregation and incidence of disease. Plant Pathol. 41:657-660.

Hughes, G., and Madden, L. V. 1993. Using the beta-binomial distribution to describe aggregated patterns of disease incidence. Phytopathology 83: 759-763.

Hughes, G., and Madden, L. V. 1995. Some methods allowing for aggregated patterns of disease incidence in the analysis of data from designed experiments. Plant Pathol. 44:927-943.

Hughes, G., and Madden, L. V. 1998. Comment: Using spatial and temporal patterns of Armillaria root disease to formulate management recommendations for Ontario's black spruce (Picea mariana) seed orchards. Can. J. For. Res. 28:154-158.

Hughes, G., Madden, L. V., and Munkvold, G. P. 1996. Cluster sampling for disease incidence data. Phytopathology 86:132-137.

Hughes, G., McRoberts, N., Madden, L. V., and Gottwald, T. R. 1997a. Relationships between disease incidence at two levels in a spatial hierarchy. Phytopathology 87:542-550.

Hughes, G., McRoberts, N., Madden, L. V., and Nelson, S. C. 1997b. Validating mathematical models of plant disease progress in space and time. IMA J. Math. Appl. Med. Biol. 14:85-112.

Hughes, G., Ramos Monreal, A., and Codesal Vara, P. 1989. Characterizing the effect of spatially heterogeneous pest injury on crop yields. Crop Res. 29:37-50.

Humeau, L., Roumagnac, P., Picard, Y., Robène-Soustrade, I., Chiroleu, F., Gagnevin, L., and Pruvost, O. 2006. Quantitative and molecular epidemiology of bacterial blight of onion in seed production fields. Phytopathology 96:1345-1354

Jeger, M. J., Stevenson, K. L., and Madden, L. V. 2017. Plant disease epidemiology. Online publication. Oxford Bibliographies.

Jones, S. J., Gent, D. H., Pethybridge, S. J., and Hay, F. S. 2011. Spatial characteristics of white mould epidemics and the development of sequential sampling plans in Australian bean fields. Plant Pathol. 60:1169-1182.

Jørgensen, B. 1997. The Theory of Exponential Dispersion Models. Chapman \& Hall, London.

Kendal, W. S. 2004. Taylor's ecological power law as a consequence of scale invariant exponential dispersion models. Ecol. Complex. 1:193-209.

Kendal, W. S., and Jørgensen, B. 2011. Taylor's power law and fluctuation scaling explained by a central-limit convergence. Phys. Rev. E 83:066115.

Keske, C., May-De Mio, L. L., and Amorim, L. 2013. Spatial pattern of brown rot within peach trees related to inoculum of Monilinia fructicola in organic orchard. J. Plant Pathol. 95:67-73.

Kriss, A. B., Paul, P. A., and Madden, L. V. 2012. Characterizing heterogeneity of disease incidence in a spatial hierarchy: A case study from a decade of observations of Fusarium head blight of wheat. Phytopathology 102:867-877.

Laranjeira, F. F., Barbosa, C. J., Santos Filho, H. P., Gonçalves, T. F., and Nickel, O. 2006. Progress, spread and natural transmission of Bahia bark scaling of citrus in Brazil. Ann. Appl. Biol. 148:187-195.

Li, B., Madden, L. V., and Xu, X.-M. 2012. Spatial analysis by distance indices: An alternative local clustering index for studying spatial patterns. Methods Ecol. Evol. 3:368-377.

Li, B. H., Yang, J. R., Li, B. D., and Xu, X. M. 2007. Incidence-density relationship of pear scab (Venturia nashicola) on fruits and leaves. Plant Pathol. 56:120-127.

Macedo, M. A., Costa, T. M., Barbosa, J. C., Pereira, J. L., Michereff Filho, M., Gilbertson, R. L., Inoue-Nagat, A. K., and Bergamin Filho, A. 2016. Temporal and spatial dynamics of begomovirus disease in tomatoes in central Brazil. Plant Pathol. 66:529-538.
Madden, L. V. 1989. Dynamic nature of within-in field disease and pathogen distributions. Pages 96-126 in: The Spatial Component of Plant Disease Epidemics. M. J. Jeger, ed. Prentice-Hall, Englewood Cliffs, NJ.

Madden, L. V., and Hughes, G. 1995. Plant disease incidence: Distributions, heterogeneity, and temporal analysis. Annu. Rev. Phytopathol. 33:529-564.

Madden, L. V., and Hughes, G. 1999a. An effective sample size for predicting plant disease incidence in a spatial hierarchy. Phytopathology 89:770-781.

Madden, L. V., and Hughes, G. 1999b. Sampling for plant disease incidence. Phytopathology 89:1088-1103.

Madden, L. V., Hughes, G., and Ellis, M. A. 1995a. Spatial heterogeneity of the incidence of grape downy mildew. Phytopathology 85:269-275.

Madden, L. V., Hughes, G., and Munkvold, G. P. 1996. Plant disease incidence: Inverse sampling, sequential sampling, and confidence intervals when observed mean incidence is zero. Crop Prot. 15:621-632.

Madden, L. V., Hughes, G., and van den Bosch, F. 2007. The Study of Plant Disease Epidemics. American Phytopathological Society Press, St. Paul, MN.

Madden, L. V., Nault, L. R., Murral, D. J., and Apelt, M. R. 1995b. Spatial pattern analysis of the incidence of aster yellows disease in lettuce. Res. Popul. Ecol. (Kyoto) 37:279-289.

Madden, L. V., Pirone, T. P., and Raccah, B. 1987. Analysis of spatial patterns of virus-diseased tobacco plants. Phytopathology 77:1409-1417.

Madden, L. V., Turechek, W. W., and Nita, M. 2002. Evaluation of generalized linear mixed models for analyzing disease incidence data obtained in designed experiments. Plant Dis. 86:316-325.

Moreira, A. S., Bergamin Filho, A., and Marques Rezende, J. A. 2015. Comparative epidemiology of three virus diseases on zucchini squash. J. Phytopathol. 163:386-394.

Moreira, A. S., Cônsoli, R., Barbosa, J. C., and Rezende, J. A. M. 2014. Spatiotemporal dynamics of zucchini squash lethal chlorosis and its relationship with the Frankliniella zucchini population. J. Phytopathol. 162: 481-490.

Nault, B. A., and Kennedy, G. G. 1996. Sequential sampling plans for use in timing insecticide applications for control of European corn borer (Lepidoptera: Pyralidae) in potato. J. Econ. Entomol. 89:1468-1476.

Navas-Cortés, J. A., Landa, B. B., Mercado-Blanco, J., Trapero-Casas, J. L., Rodríguez-Jurado, D., and Jiménez-Díaz, R. M. 2008. Spatiotemporal analysis of spread of infections by Verticillium dahliae pathotypes within a high tree density olive orchard in southern Spain. Phytopathology 98:167-180.

Nita, M., Ellis, M. A., and Madden, L. V. 2008. Variation in disease incidence of Phomopsis cane and leaf spot of grape in commercial vineyards in Ohio. Plant Dis. 92:1053-1061.

Nita, M., Ellis, M. A., and Madden, L. V. 2012. Spatial pattern of Phomopsis cane and leaf spot symptoms in commercial vineyards in Ohio. J. Phytopathol. 160:26-36.

Park, B., and Park, H. 2008. Use of pseudo-likelihood estimation in Taylor's power law with correlated responses. Commun. Korean Stat. Soc. 15: 993-1002.

Patil, G. P., and Stiteler, W. M. 1974. Concepts of aggregation and their quantification: A critical review with some new results and applications. Res. Popul. Ecol. 15:238-254.

Perry, J. N. 1987. Iterative improvement of a power transformation to stabilize variance. Appl. Stat. 36:15-21.

Perry, J. N. 1988. Some models for spatial variability of animal species. Oikos 51:124-130

Perry, J. N. 1994. Chaotic dynamics can generate Taylor's power law. Proc. R. Soc. Lond. B 257:221-226.

Perry, J. N., and Taylor, L. R. 1985. Adès: New ecological families of speciesspecify frequency distributions that describe repeated spatial samples with an intrinsic power-law variance-mean property. J. Anim. Ecol. 54:931-953.

Perry, J. N., and Woiwod, I. P. 1992. Fitting Taylor's power law. Oikos 65: 538-542.

Pethybridge, S. J., Hay, F. S., and Gent, D. H. 2010. Characterization of the spatiotemporal attributes of Sclerotinia flower blight epidemics in a perennial pyrethrum pathosystem. Plant Dis. 94:1305-1313.

Piepho, H.-P. 1999. Analysing disease incidence data from design experiments by generalized linear mixed models. Plant Pathol. 48:668-674.

Piepho, H.-P. 2003. The folded exponential transformation for proportions. Statistician 52:575-589.

Prentice, R. L. 1986. Binary regression using an extended beta-binomial distribution, with discussion of correlation induced by covariate measurement errors. J. Am. Stat. Assoc. 81:321-327.

Rappussi, M. C. C., Eckstein, B., Flôres, D., Haas, I. C. R., Amorim, L., and Bedendo, I. P. 2012. Cauliflower stunt associated with a phytoplasma of subgroup 16SrIII-J and the spatial pattern of disease. Eur. J. Plant Pathol. 133:829-840.

Ridout, M. S., Demetrio, C. G. B., and Firth, D. 1999. Estimating intra-class correlation for binary data. Biometrics 55:137-148. 
Ridout, M. S., and Xu, X.-M. 2000. Relationships between several quadratbased statistical measures used to characterize spatial aspects of disease incidence data. Phytopathology 90:568-575.

Rosenberg, M., and Anderson, C. 2016. Spatial pattern analysis. Online publication. Oxford Bibliographies. D. Gibson, ed.

Roumagnac, P., Pruvost, O., Chiroleu, F., and Hughes, G. 2004. Spatial and temporal analyses of bacterial blight of onion caused by Xanthomonas axonopodis pv. allii. Phytopathology 94:138-146.

Savary, S., Castilla, N. P., and Willocquet, L. 2001. Analysis of the spatiotemporal structure of rice sheath blight epidemics in a farmer's field. Plant Pathol. 50:53-68.

Schabenberger, O., and Pierce, F. J. 2002. Contemporary Statistical Models for the Plant and Soil Sciences. CRC Press, Boca Raton, FL.

Scott, J. B., Gent, D. H., Pethybridge, S. J., and Hay, F. S. 2014. Spatiotemporal characterization of Sclerotinia crown rot epidemics in pyrethrum. Plant Dis. 98:267-274.

Seber, G. A. F., and Wild, C. J. 2003. Nonlinear Regression. John Wiley \& Sons, New York.

Shah, D. A., and Bergstrom, G. C. 2002. A rainfall-based model for predicting the regional incidence of wheat seed infection by Stagonospora nodorum in New York. Phytopathology 92:511-518.

Shah, D. A., Bergstrom, G. C., and Ueng, P. P. 2001. Foci of Stagonospora nodorum blotch in winter wheat before canopy development. Phytopathology 91:642-647.

Shah, D. A., Clear, R. M., Madden, L. V., and Bergstrom, G. C. 2002. Summarizing the regional incidence of seed-borne fungi with the $\beta$-binomial distribution. Can. J. Plant Pathol. 24:168-175.

Shah, D. A., Dillard, H. R., and Nault, B. A. 2005a. Sampling for the incidence of aphid-transmitted viruses in snap bean. Phytopathology 95:1405-1411.

Shah, D. A., Dillard, H. R., and Pethybridge, S. J. 2018. Hierarchical models for white mould in snap bean. Plant Pathol. 67:145-155.

Shah, D. A., Pucci, N., and Infantino, A. 2005b. Regional and varietal differences in the risk of wheat seed infection by fungal species associated with Fusarium head blight in Italy. Eur. J. Plant Pathol. 112:13-21.

Shi, P.-J., Sandhu, H. S., and Reddy, G. V. P. 2016. Dispersal distance determines the exponent of the spatial Taylor's power law. Ecol. Modell. 335:48-53.

Shiyomi, M., Takahashi, S., Yoshimura, J., Yasuda, T., Tsutsumi, M., Tsuiki, M., and Hori, Y. 2001. Spatial heterogeneity in a grassland community: Use of power law. Ecol. Res. 16:487-495.

Silva Junior, G. J., Spósito, M. B., Marin, D. R. Ribeiro, P. J., Jr., and Amorim, L. 2014. Spatiotemporal characterization of citrus postbloom fruit drop in Brazil and its relationship to pathogen dispersal. Plant Pathol. 63:519-529.

Smith, D. M. 1983. Maximum likelihood estimation of the parameters of the beta binomial distribution. Appl. Stat. 32:196-204.

Smith, R. J. 2009. Use and misuse of the reduced major axis for line-fitting. Am. J. Phys. Anthropol. 140:476-486.

Sokal, R. R., and Rohlf, F. J. 1995. Biometry, 3rd ed. Freeman, New York.

Southwood, T. R. E. 1966. Ecological Methods. Methuen \& Co., London.

Spolti, P., Shah, D. A., Fernandes, J. M. C., Bergstrom, G. C., and Del Ponte, E. M. 2015. Disease risk, spatial patterns, and incidence-severity relationships of Fusarium head blight in no-till spring wheat following maize or soybean. Plant Dis. 99:1360-1366.

Spósito, M. B., Amorim, L., Bassanezi, R. B., and Hau, B. 2008. Spatial pattern of black spot incidence within citrus trees related to disease severity and pathogen dispersal. Plant Pathol. 57:103-108.

Stroup, W. W. 2013. Generalized Linear Mixed Models: Modern Concepts, Methods and Applications. CRC Press, Boca Raton, FL.

Sun, P., and Madden, L. V. 1997. Using a normal approximation to test for the binomial distribution. Biom. J. 39:533-544.

Taylor, L. R. 1961. Aggregation, variance, and mean. Nature 189:732-735.
Taylor, L. R., Perry, J. N., Woiwod, I. P., and Taylor, R. A. J. 1988. Specificity of the spatial power-law exponent in ecology and agriculture. Nature 332: 721-722.

Taylor, L. R., and Taylor, R. A. J. 1977. Aggregation, migration and population mechanics. Nature 265:415-421.

Taylor, L. R., Taylor, R. A. J., Woiwod, I. P., and Perry, J. N. 1983. Behavioural dynamics. Nature 303:801-804.

Taylor, L. R., and Woiwod, I. P. 1982. Comparative dynamics I. Relationships between inter- and intra-specific spatial and temporal variance/mean population parameters. J. Anim. Ecol. 51:879-906.

Tsuiki, M., Wang, Y. S., Tsutsumi, M., and Shiyomi, M. 2005. Analysis of grassland vegetation of the Southwest Heilongjiang steppe (China) using the power law. J. Integr. Plant Biol. 47:917-926.

Turechek, W. W., Ellis, M. A., and Madden, L. V. 2001. Sequential sampling for incidence of Phomopsis leaf blight of strawberry. Phytopathology 91:336-347.

Turechek, W. W., and Madden, L. V. 1999a. Spatial pattern analysis of strawberry leaf blight in perennial production systems. Phytopathology 89:421-433.

Turechek, W. W., and Madden, L. V. 1999b. Spatial pattern analysis and sequential sampling for the incidence of leaf spot on strawberry in Ohio. Plant Dis. 83:992-1000.

Turechek, W. W., and Madden, L. V. 2000. Analysis of the association between the incidence of two spatially aggregated foliar diseases of strawberry. Phytopathology 90:157-170.

Turechek, W. W., and Madden, L. V. 2001. Effect of scale on plant disease incidence and heterogeneity in a spatial hierarchy. Ecol. Modell. 144:77-95.

Turechek, W. W., and Madden, L. V. 2003. A generalized linear modeling approach for characterizing disease incidence in a spatial hierarchy. Phytopathology 93:458-466.

Turechek, W. W., Madden, L. V., Gent, D. H., and Xu, X.-M. 2011. Comments regarding the binary power law for heterogeneity of disease incidence. Phytopathology 101:1396-1407.

Turechek, W. W., and Mahaffee, W. F. 2004. Spatial pattern analysis of hop powdery mildew in the Pacific Northwest: Implications for sampling. Phytopathology 94:1116-1128.

Turechek, W. W., and McRoberts, N. 2013. Considerations of scale in the analysis of spatial pattern of plant disease epidemics. Annu. Rev. Phytopathol. 51:453-472.

Turechek, W. W., Roberts, P. D., Stansly, P. A., Webster, C. G., Kousik, C. S., and Adkins, S. 2014. Spatial and temporal analysis of Squash vein yellowing virus infections in watermelon. Plant Dis. 98:1671-1680.

Van der Heyden, H., Lefebvre, M., Roberge, L., Brodeur, L., and Carisse, O. 2014. Spatial pattern of strawberry powdery mildew (Podosphaera aphanis) and airborne inoculum. Plant Dis. 98:43-54.

Warton, D. I., and Hui, F. K. C. 2011. The arcsine is asinine: The analysis of proportions in ecology. Ecology 92:3-10.

$\mathrm{Xu}, \mathrm{X}$., and Madden, L. V. 2002. Incidence and density relationships of powdery mildew on apple. Phytopathology 92:1005-1014.

Xu, X.-M., and Madden, L. V. 2014. The limits of the binary power law describing spatial variability for incidence data. Plant Pathol. 63:973-982.

Xu, X.-M., and Ridout, M. S. 1998. Effects of initial epidemic conditions, sporulation rate, and spore dispersal gradient on the spatio-temporal dynamics of plant disease epidemics. Phytopathology 88:1000-1012.

Xu, X.-M., and Ridout, M. S. 2000. Effects of quadrat size and shape, initial epidemic conditions, and spore dispersal gradient on spatial statistics of plant disease epidemics. Phytopathology 90:738-750.

Yohai, V. J. 1987. High breakdown point and high efficiency robust estimates for regression. Ann. Stat. 15:642-656.

Yu, G. 2009. Variance stabilizing transformations of Poisson, binomial and negative binomial distributions. Stat. Probab. Lett. 79:1621-1629. 